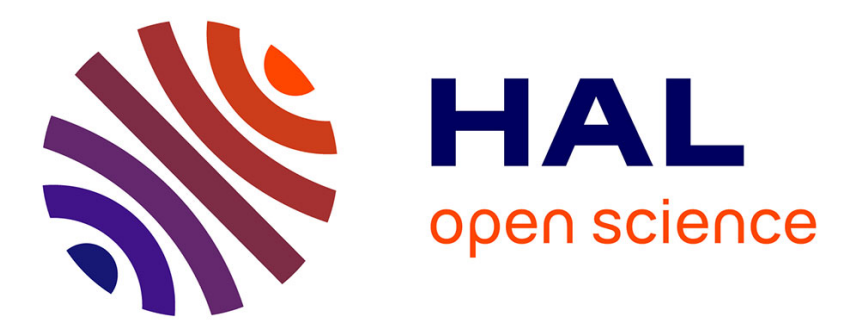

\title{
Les biens et les revenus publics dans le royaume d'Italie (jusqu'au milieu du Xe siècle)
}

François Bougard

\section{To cite this version:}

François Bougard. Les biens et les revenus publics dans le royaume d'Italie (jusqu'au milieu du Xe siècle). Biens publics, biens du roi. Les bases économiques des pouvoirs royaux dans le haut Moyen Âge - Beni pubblici, beni del re. Le basi economiche dei poteri regi nell'alto medioevo, Oct 2016, Roma, Italie. pp.79-120. halshs-02426409

\section{HAL Id: halshs-02426409 \\ https://shs.hal.science/halshs-02426409}

Submitted on 16 Jul 2021

HAL is a multi-disciplinary open access archive for the deposit and dissemination of scientific research documents, whether they are published or not. The documents may come from teaching and research institutions in France or abroad, or from public or private research centers.
L'archive ouverte pluridisciplinaire HAL, est destinée au dépôt et à la diffusion de documents scientifiques de niveau recherche, publiés ou non, émanant des établissements d'enseignement et de recherche français ou étrangers, des laboratoires publics ou privés. 
[paru dans : Biens publics, biens du roi. Les bases économiques des pouvoirs royaux dans le haut Moyen Âge - Beni pubblici, beni del re. Le basi economiche dei poteri regi nell'alto medioevo, éd. F. Bougard et Vito Loré, Turnhout, Brepols, 2019 (Seminari del Centro interuniversitario di storia e archeologia dell'alto medioevo, 9), p. 79-120.]

\section{François Bougard}

Les biens et les revenus publics dans le royaume d'Italie (jusqu'au milieu du $\mathrm{X}^{\mathrm{e}}$ siècle)

I. La période lombarde. - II. La mise en place du régime carolingien. - III. Tableau des biens publics au $\mathrm{IX}^{\mathrm{e}}$ siècle. - La fin du IX ${ }^{\mathrm{e}}$ et la première moitié $\mathrm{du} \mathrm{X}^{\mathrm{e}}$ siècle : des tensions à la reprise en main par Hugues de Provence.

La définition des regalia promulguée en 1158 par l'assemblée de Roncaglia, destinée à clarifier une situation depuis longtemps confuse, permet de se faire une idée du chemin qui sépare la fin de l'Antiquité et le Moyen Âge en matière de biens et de revenus publics en Italie ${ }^{1}$. L'énumération de ce que Frédéric Barberousse prétend revenir à l'Empire repose pour l'essentiel sur des prélèvements indirects : le produit des amendes et les droits liés aux activités commerciales. Des éléments fonciers sont aussi présents : arimanniae, voies publiques, cours d'eau navigables, pêcheries et salines, mines d'argent, biens tombés dans le domaine public pour cause de justice ou bien parce qu'ils sont devenus vacants par défaut d'héritier, palais urbains - dont traitait plus précisément la loi Palatia et pretoria, perdue. Chaque entrée de cette liste pourrait être cochée dès le $\mathrm{IX}^{\mathrm{e}}$ siècle, sinon avant. Cependant, il n'y figure pas d'exploitation rurale, de curtis regia. Pour en trouver, il faut se reporter à la liste des vingt-huit curię de Lombardia censées pourvoir à la mensa du roi des Romains : un texte rédigé quelques années auparavant, antérieurement à la descente de Frédéric Barberousse en Italie et dont Renato Bordone a montré l'hétérogénéité. Issu du collage de plusieurs pièces de dates différentes, il fournit des informations non vérifiées et non localisées par le compilateur, qui prend soin de dire son ignorance : « combien elles (les curię) donnent en réalité, nul ne peut le savoir, ni faire la recherche avant que nous n'allions en Lombardie $»^{2}$. Le troisième pilier des revenus impériaux est celui qu'exprime la loi Tributum dabatur pro capite, tributum dabatur pro agro, dont le seul incipit, à l'imparfait, révèle le côté fantasmé : il est question du passé romain, et nullement des réalités de la perception fiscale du milieu du XII ${ }^{\mathrm{e}}$ siècle.

Si l'on en jugeait par ces seules sources, nous aurions donc : des revenus principalement indirects ; des biens royaux très mal connus de l'autorité elle-même ; un projet d'impôt direct fondé sur un passé mythique. Vu de très haut, tout se passe comme si, entre le haut Moyen Âge et le temps de Frédéric Barberousse, les plateaux de la balance s'étaient inversés, avec une importance toujours plus grande accordée au prélèvement indirect et une part toujours plus faible de la rente foncière, devenue insaisissable au XII ${ }^{\mathrm{e}}$ siècle. Sur la longue durée, l'emprise foncière de la royauté ou du publicum aurait aussi subi un mouvement de baisse inexorable, que l'on a tenté de quantifier il y a déjà longtemps. En 1896, Paul Darmstädter évaluait les pertes comme suit, pour la Lombardie et le Piémont : $5 \%$ pour la période lombarde, $27 \%$ pour les temps carolingiens (774-887), $41 \%$ pour celui des rois dits nationaux (887-962), $18 \%$ entre

\footnotetext{
${ }^{1}$ MGH Const. I, $n^{\circ} 175$, p. 244-245. C. Brühl, « Die Finanzpolitik Friedrich Barbarossas in Italien » [1971], in Id., Aus Mittelalter und Diplomatik. Gesammelte Aufsätze, I, Hildesheim, 1989, p. [13-37] 267-291.

2 C. Brühl, T. Kölzer, Das Tafelgüterverzeichnis des römischen Königs, Cologne-Vienne, 1979, pl. IV; R. Bordone, «L'enigmatico elenco dei beni fiscali 'in Lombardia' al tempo di Federico Barbarossa. Alcune proposte interpretative », in M. Bassetti et al. (éd.), Studi sul Medioevo per Andrea Castagnetti, Bologne, 2011, p. $59-73$.
} 
962 et $1024,9 \%$ de 1024 à $1093^{3}$. Une telle précision, et la tentative même du comptage peuvent faire sourire, mais le constat est bien celui d'un siphonnage constant, plus ou moins rapide selon les époques, par le biais du don ou de la «privatisation » / «appropriation » / « patrimonialisation ». On en trouve au reste confirmation dans le fait que, passées les années 1030, les mentions de terra regis dans les descriptions de confins fournies par la documentation privée se font plus rares qu'auparavant. Mais si le phénomène est bien connu, il reste difficile d'en rendre compte autrement que dans les termes peu satisfaisants de «l'appauvrissement» du fisc. La difficulté vient de ce qu'on ne connaît guère les choses que du côté de la dépense, et très peu du côté de la recette. Ou plutôt : on voit les sorties du côté de l'immobilier, les entrées du côté de l'impôt indirect; d'où une appréciation biaisée de part et d'autre. Les lignes qui suivent ne prétendent pas apporter du neuf, mais rappeler, au moment où les enquêtes sur la question fiscale se multiplient, les grands traits d'une évolution.

Les informations sur la période lombarde, déjà amplement examinées par d'autres ${ }^{4}$, peuvent être présentées rapidement. Le fisc lombard, constitué à mesure de la prise en main de l'Italie romano-byzantine, s'appuie : 1) sur l'héritage impérial, qu'avait maintenu le régime ostrogothique ; 2) sur les dépouilles des grands propriétaires (éliminés physiquement, exilés ou absents de longue date) ; 3) sur les biens des églises épiscopales dès lors que celles-ci n'ont pas trouvé grâce aux yeux des nouveaux maîtres du sol, à la différence de cités comme Trévise ${ }^{5}$. Il n'est pas structuré de la manière qu'au nord des Alpes puisque, si l'on en croit Paul Diacre, il $\mathrm{y}$ eut après le règne d'Alboin et celui de Cleph un temps de gouvernement exclusivement ducal (574-584), suivi de l'élection d'Authari : la restauratio du royaume fut alors accompagnée de l'affectation aux regales usus de la moitié des substantiae des ducs, «afin de dégager de quoi subvenir au roi lui-même, à son entourage et à ceux qui étaient à son service dans les différents emplois $»^{6}$. Soit quatre bénéficiaires des biens publics : les ducs, le roi et, à travers lui, sa suite et ses partisans répartis entre les «offices »-à la cour et/ou sur le territoire, on ne sait trop. D'où, aussi, une position foncière des ducs forte dès l'origine et une moindre hégémonie du roi.

Cependant, quel que soit le crédit qu'on veuille accorder à la narration de Paul Diacre à propos d'une situation antérieure de deux siècles à l'écriture de son ouvrage, il serait anachronique de vouloir la faire coller aux réalités que laissent entrevoir les actes du VIII ${ }^{\mathrm{e}}$ siècle. À cette date, l'importance constatée du fisc ducal dans les duchés de Spolète et de Bénévent tient plus vraisemblablement à l'autonomie politique croissante des deux régions depuis la fin $\mathrm{du} \mathrm{VI}^{\mathrm{e}}$ siècle. Elle a mené les ducs à avoir leur propre production de diplômes, à la datation privée de référence à l'autorité royale de manière systématique à Bénévent et jusqu'aux années 760 à Spolète, et à disposer de la terre publique selon leur bon vouloir. Par contraste, la position

\footnotetext{
${ }^{3}$ P. Darmstädter, Das Reichsgut in der Lombardei und Piemont (568-1250), Strasbourg, 1896, réimpr. Berlin, 1965, p. 5. Le total est de $100 \%$, ce qui ne signifie pas, bien sûr, que l'intégralité des biens publics avait disparu à la fin $\mathrm{du} \mathrm{XI}^{\mathrm{e}}$ siècle.

${ }^{4}$ Voir surtout S. Gasparri, «Le basi economiche del potere pubblico in età longobarda », in P. C. Díaz et I. M. Viso (éd.), Between Taxation and Rent. Fiscal Problems from Late Antiquity to Early Middle Ages, Bari, 2011, p. 71-85; auparavant, Id., «Il regno longobardo in Italia. Struttura e funzionamento di uno stato altomedievale », in Id. (éd.), Il regno dei Longobardi in Italia. Archeologia, società, istituzioni, Spolète, 2004, p. 1-92: p. ***; C. Brühl, Fodrum, Gistum, Servitium regis. Studien zu den wirtschaftlichen Grundlagen des Königtums im Frankreich und in den fränkischen Nachfolgestaaten Deutschland, Frankreich und Italien vom 6. bis zur Mitte des 14. Jahrhunderts, Cologne-Graz, 1968 (Kölner historische Abhandlungen, 14/1), p. 357-368, 375-392.

${ }^{5}$ Paul Diacre, Historia Longobardorum II, 12, éd. G. Waitz, MGH SSrL, p. 79.

${ }^{6}$ Historia Langobardorum. III, 16, p. 101 : ob restaurationem regni duces qui tunc erant omnem substantiarum suarum medietatem regalibus usibus tribuunt, ut esse possit, unde rex ipse sive qui ei adhaererent eiusque obsequiis per diversa officia dediti alerentur.
} 
du roi est beaucoup plus forte en plaine du Pô, où il a largement rattrapé son retard entre $\mathrm{VI}^{\mathrm{e}}$ et $\mathrm{VIII}^{\mathrm{e}}$ siècle. Il est le seul acteur en mesure de gérer les ressources publiques tout en en laissant des traces écrites.

Dans les années 760-770, les diplômes pour S. Salvatore de Brescia et pour S. Maria de Sesto montrent que le roi a la main sur des biens relevant aussi bien de «sa »curtis publica que de la curtis ducale - qu'il dit aussi volontiers sienne - à Brescia et à Trévise, de même que de biens de l'une ou de l'autre ${ }^{7}$. Cette assimilation au moins partielle entre les deux entités tient probablement à leur contiguïté physique : c'est la seule trace que l'on pourrait invoquer du partage originel des ressources. Au nord du royaume, c'est au reste la seule mention de biens ducaux avant celles des $\mathrm{IX}^{\mathrm{e}}-\mathrm{X}^{\mathrm{e}}$ siècles, qui ressortent d'un autre cadre institutionnel.

Instance supérieure, la curtis regia relève de la potestas du souverain, assimilée à la potestas publica ou «au palais », ou encore à la potestas palatii nostri, toutes formulations équivalentes $^{8}$. Comme telle, elle est urbaine ${ }^{9}$, au point que civitas et curtis regia paraissent parfois interchangeables, ce qui n'empêche pas la présence de multiples curtes regiae en milieu rural. Quelles qu'elles soient, ce sont à elles que sont rattachés les gualdi, espaces sylvopastoraux : on parle de gualdus publicus ou noster, de pascua publica, de publica animalia ${ }^{10}$. La présence foncière du roi est ainsi double, à la fois au cœur du territoire et sur ses marges entendues au sens géographique et non économique -, et avec des modes d'exploitation que l'on pressent différents.

Le souci de disposer d'une information précise et à jour sur les biens publics est notable : témoins la demande faite aux actores regis d'établir des inventaires (brevia) pour l'ensemble des curtes royales en 733 et le breve du gagius de Reggio rédigé de la main de son responsable, le waldeman Abo, vers $770^{11}$. Relevons toutefois que les brefs dont il s'agit sont semble-tdestinés à une information d'ordre territorial, pour une meilleure connaissance juridique nécessaire pour contrecarrer les malversations aux dépens de la chose publique -, et ne relèvent pas d'une préoccupation d'ordre gestionnaire, économique. Les biens publics bénéficient par ailleurs d'une protection juridique particulière, avec la prescription de soixante ans et non de trente comme pour ceux des personnes privées, et une composition deux fois plus élevée ${ }^{12}$. Rappelons enfin que leur étendue a fait l'objet d'une estimation par Paul Darmstädter pour le Piémont et la Lombardie : un neuvième du sol, chiffre repris par Carlrichard Brühl (10\%) sans autre examen, mais qui relève bien sûr de l'hypothèse ${ }^{13}$.

La terre publique est créatrice de ressources, puisque son usage est soumis à un versement de droits, comme ceux que payaient les abbayes de Farfa et de S. Salvatore de

\footnotetext{
${ }^{7}$ Codice diplomatico longobardo, III, éd. C. Brühl, Rome, 1973 (Fonti per la storia d'Italia, 64), no 33, a. 765, donation à. S. Salvatore de Brescia d'un casale, pertinens ad curtem nostram et ad curtem ducalem; $\mathrm{n}^{\circ} 39$, a. 767, donation à S. Salvatore de deux moulins, sicuti ad curte nostri publica vel ad curtem ducalem pertinuit; $\mathrm{n}^{\circ} 44$, a. 772: confirmation à S. Salvatore de biens ex iura curtis nostrae regiae que et ex iura curtis docalis; p. 304 , diplôme d'Adelchis perdu pour S. Maria de Sesto, lui attribuant la contribution en sel et en porcs qu'un vicus versait habituellement in palacia nostro seu in curte ducali nostra Tarvisana. Brühl, Fodrum, p. 365.

${ }^{8}$ Codice diplomatico longobardo, III, $\mathrm{n}^{\circ} 18$, a. 744 : palatium nostrum ; ${ }^{\text {os }} 31$ et 39, a. 759, 767: nostra potestas ; $\mathrm{n}^{\circ} 41$, a. 772 : potestas nostra vel palacium nostrum ; $\mathrm{n}^{\circ} 44$, a. 772 : potestas palatii nostri, potestas publica. Gasparri, «Il regno longobardo », p. 21-22.

${ }^{9}$ Outre la curtis regia de Brescia déjà mentionnée, voir Codice diplomatico longobardo, III, $\mathrm{n}^{\circ}$ 6, a. 674 : litige entre la curtis regia de Plaisance et celle de Parme ; Codice diplomatico longobardo, I, éd. L. Schiaparelli, Rome, 1929 (Fonti per la storia d'Italia, 62), n ${ }^{\circ} 17$, a.. 714 : curtis regia d'Arezzo; $\mathrm{n}^{\circ} 19$, a. $715:$ c.r. de Sienne ; $\mathrm{n}^{\circ} 113$, a. 754 (= Chartae latinae antiquiores [ChLA], XXXII, éd. G. Nicolaj, Dietikon-Zurich, 1989, $\mathrm{n}^{\circ}$ 942) : c.r. de Lucques. Brühl, Fodrum, p. 357-358 ; Gasparri, Il regno longobardo, p. 22-34; Id., «Le basi economiche », p. 7980.

${ }^{10}$ Codice diplomatico longobardo, III, $\mathrm{n}^{\circ}$ 24, a. 749-751 (Modène), $\mathrm{n}^{\text {os }} 28$ et 43, a. 756, 770-772 (Spolète, Rieti), $\mathrm{n}^{\circ}$ 41, a. 772 (Reggio). Gasparri, «Il regno longobardo », p. 28.

${ }^{11}$ Notitia de actoribus regis, 5 ; Codice diplomatico longobardo, III, $\mathrm{n}^{\circ} 41$.

${ }^{12}$ Liutprand, 78 ; Notitia de actoribus regis, 2 ; Ratchis, 6.

${ }^{13}$ Darmstädter, Reichsgut, p. 5 ; Brühl, Fodrum, p. 383.
} 
Brescia sur les pâturages (daticum, herbaticum, scaticum) avant d'en être exemptés vers 770, comme l'avaient été avant elles plusieurs habitants de Pegognaga, près de Mantoue, pour la forêt de Fiesso ${ }^{14}$. La contribution en sel et en porcs fournie au palais et/ou à la curtis ducale de Trévise par le vicus de Piove di Sacco est probablement aussi une redevance issue de l'usage de la terre fiscale ${ }^{15}$. Un autre exemple est fourni par le fictum versé par un groupe de parents à la curtis royale - un centre d'exploitation proche, ou bien la curtis de Plaisance - au titre de l'exploitation de biens dans le secteur collinaire préapenninique au sud de Plaisance, en 770 : au moment d'échanger ces terres avec un autre de leurs parents, ils s'engagent à verser le fictum à sa place ${ }^{16}$. Deux interprétations sont ici possibles. Ou bien les terres assujetties à un fictum public sont possédées en toute propriété, ce qui impliquerait qu'elles ont un statut particulier, transmis au fil des générations, en vertu duquel elles sont redevables envers le fisc. La comparaison s'imposerait alors avec celles sur lesquelles, en contexte byzantin, pèsent des obligations militaires. Ou bien, et plus simplement, il s'agit de biens concédés sur l'espace public, certes librement transmissibles mais dont l'usage est grevé d'une taxe. L'engagement à payer le fictum dans l'acte de 770 est alors une simple assurance que, au moment de la transaction, l'autre partie ne reçoive pas un terrain pour lequel il devra payer dans un futur proche; dans l'avenir, c'est le nouvel exploitant, en revanche, qui s'en acquittera ${ }^{17}$. Cette deuxième hypothèse est d'autant plus probable que cette clause suit celle, habituelle, de defensio de l'acte par son auctor juridique : manière de dire que, en cas de contestation de la transaction, et si celle-ci devait être annulée, le nouvel entrant ne serait pas perdant. Le texte est en tout cas précieux, en ce qu'il montre que les biens du fisc peuvent sans difficulté s'intégrer dans le jeu du marché de la terre ${ }^{18}$. Malgré tout, cela n'est pas sans risque

Si le produit de la rente foncière ou des droits d'usage alimente de manière générale la res publica, sa répartition peut donc varier, depuis le niveau local (rural ou urbain) jusqu'au versement direct au palais. Les deux ne se confondent pas, comme l'indique la Notitia de actoribus regis de 733, qui déplore le fait que les actores des curtes royales gardent pour eux le produit des amendes qu'ils infligent, en un détournement qui ne profite nec in rebus publicis nec nulla rationem palatii ${ }^{19}$. Nous aurons l'occasion de voir la pérennité d'une telle distinction ${ }^{20}$.

Les prélèvements indirects concernent également les tonlieux et taxes sur le sel dans les marchés et aux ports, attestés à partir du début du VIII ${ }^{\mathrm{e}}$ siècle d'abord pour les «étrangers » (ceux de Comacchio) puis pour les ressortissant du royaume ${ }^{21}$. Quant à la pensio de trente livres de savon fournie chaque année au Palais « depuis la cité de Plaisance » et dont le roi Ildeprand attribua le produit à l'église épiscopale, je suis tenté d'y voir la ponction sur la production des saponarii locaux, ponction que pourrait justifier le fait qu'ils détenaient un monopole, comme les savonniers de Pavie au $\mathrm{X}^{\mathrm{e}}$ siècle. Il n'est toutefois pas exclu que l'expression ex Placentina

\footnotetext{
${ }^{14}$ Codice diplomatico longobardo, III, $\mathrm{n}^{\text {os }} 43-44$ et p. 297-298.

${ }^{15}$ Codice diplomatico longobardo, III, p. 305 ; Brühl, Fodrum, p. 385 ; Gasparri, « Il regno longobardo », p. 42; Id., «Le basi economiche », p. 81.

${ }^{16}$ ChLA XXVII, éd. J.-O. Tjäder, Dietikon-Zurich, 1992, no 826 : repromettttemus nos..., ut de ipso ficto, quod in corte domno regi dare deveamus, ut nos te exinde secure facere deveamus.

${ }^{17}$ La situation est proche de celle décrite dans un capitulaire de Louis le Pieux daté de 818-819 et qui fut diffusé en Italie, à propos de la terre dite «tributaire » : l'église ou l'individu qui viendrait à entrer en possession (par le jeu du marché ou des successions) d'une terre versant « tribut » à la partie publique doit continuer à s'en acquitter, sauf exonération explicite : MGH Capit. I, n 140, p. 287.

${ }^{18}$ Gasparri, « Il regno longobardo », p. 31.

${ }^{19}$ Notitia de actoribus regis, 3, éd. et trad. C. Azzara et S. Gasparri, Le leggi dei Longobardi, Milan, 1992, p. 229230 : T. Lazzari, « La tutela del patrimonio fiscale : pratiche di salvaguardia del pubblico e autorità regia nel regno longobardo del secolo VIII », Reti Medievali Rivista, 18, 1, 2017 (http://rivista.retimedievali.it), p. [8].

${ }^{20}$ Infra, texte correspondant à la note 48.

${ }^{21}$ Codex Sicardi, doc. 2 (715 ou 730), éd. V. Leoni pour le Codice diplomatico della Lombardia medievale, 2004, http://cdlm.unipv.it/edizioni/cr/cremona-sicardo/carte/.
} 
civitate utilisée dans le diplôme se rapporte à l'ensemble de la ville, ce qui en ferait alors plutôt une taxe directe ${ }^{22}$.

$\mathrm{Au}$ VIII ${ }^{\mathrm{e}}$ siècle, il n'est bien sûr plus question, au titre de ces prélèvements directs, de la tertia pars frugum qui a pu peser au lendemain de la conquête sur ceux des «nobles romains » qui n'avaient pas été tués ${ }^{23}$. La taxation, pour autant qu'on puisse l'appeler ainsi, consiste en des réquisitions de la force de travail sous forme de scuviae (excubiae), angariae, opera et autres utilitates, pesant sur les libres. En revanche, le palais de Pavie recevait une fois l'an une contribution (censum, pensio, datio) de la part de l'ensemble du royaume: non pas des individus, mais des collectivités. C'est ce qui ressort du fait qu'en 781, au moment où Charlemagne confirma la «donation» de Pépin à la papauté, il attribua à celle-ci ladite contribution pour ce qui relevait de la Tuscie lombarde et du duché de Spolète. La confirmation prend soin de préciser qu'un tel transfert de ressources n'impliquait ni dominatio ni subiectio des territoires considérés au profit de Saint-Pierre, ce qui livre en creux la motivation de ce versement annuel et l'identité des payeurs : la reconnaissance de la souveraineté de Pavie de la part des communautés politiques ${ }^{24}$.

La redistribution se fait par le biais du don, aux églises ou aux particuliers. Ces derniers peuvent recevoir non seulement des terres, mais aussi des équipements, comme des moulins, ou encore des salines ${ }^{25}$. Que la pratique des dona regum $^{26}$ aux individus soit chose aussi courante que les dona ducum étudiés pour Spolète et Bénévent ${ }^{27}$ ressort moins des actes conservés que de leurs mentions, comme celles qui figurent dans la liste dite des « archives d'Alahis », dans les années 760 : où l'on voit que ce personnage, probablement gastald de Lucques, donc administrateur du publicum, a reçu pas moins de sept préceptes de la part du roi Liutprand, dont un destiné à en confirmer six en bloc ${ }^{28}$. Il faut aussi prendre en compte l'attribution de terres fiscales aux arimanni en échange de leur service comme exercitales: témoin la silva arimannorum située dans le Val Ceno, au sud de Plaisance, qui fut divisée en quotes-parts selon un principe dont le souvenir subsistait dans les années $820^{29}$.

Comment équilibrer le système, c'est-à-dire les sorties et les entrées ? Les dons du roi aux églises, la dotation de fondations particulièrement importantes comme S. Salvatore de Brescia, la rémunération des agents sous forme de terres, l'entretien des fidélités individuelles par le don d'objets précieux, les butins concédés ou les tributs à payer aux puissances étrangères - aux Francs, à la fin du $\mathrm{VI}^{\mathrm{e}}$ siècle et dans les années $750^{30}$ - sont des ponctions importantes.

\footnotetext{
${ }^{22}$ Codice diplomatico longobardo, III, $\mathrm{n}^{\mathrm{os}} 18,27$; C. Brühl, C. Violante, Die “Honorantiae civitatis Papie”. Transkription, Edition, Kommentar, Köln-Wien, 1983, p. 23 § 13 ; infra, texte correspondant à la note 151. P. Racine, «Associations de marchands et associations de métiers en Italie de 600 à 1200 », dans B. Schwineköper (éd.), Gilden und Zünfte. Kaufmännische und gewerbliche Genossenschaften im frühen und hohen Mittelalter, Sigmaringen, 1985 (Vorträge und Forschungen, 29), p. 127-149 ; Gasparri, «Il regno longobardo », p. 35; Id., «Le basi economiche », p. 83-84.

${ }^{23}$ Paul Diacre, Historia Langobardorum II, 32, MGH SSrL, p. 90.

${ }^{24}$ DD L I 125, p. 318 1. 18-20 ; Gasparri, Il regno longobardo, p. 36 ; Id., «Le basi economiche », p. 84.

${ }^{25}$ Codice diplomatico longobardo, III, $\mathrm{n}^{\mathrm{o}} 15$ et p. 267-270, 283-286, 294-296, 304-308, 311.

${ }^{26}$ Selon l'expression de Codice diplomatico longobardo, III, $\mathrm{n}^{\circ} 42$, p. 2451.11 à propos de l'origine des biens transmis par un particulier à sa femme et sa fille : quantum habere visus fuerat de dona regum seu de iura parentum quam eciam de comparacione.

${ }^{27}$ S. Collavini, « Duchi e società locali nei ducati di Spoleto e di Benevento nel secolo VIII », in I Longobardi nei ducati di Spoleto e di Benevento. Atti del XVI Congresso internazionale di studi sull'alto medioevo, Spolète, 2003, p. $125-166$.

${ }^{28}$ Codice diplomatico longobardo, III, p. 267-268; A. Ghignoli, « Su due famosi documenti pisani dell’VIII secolo », Bullettino dell'Istituto storico italiano per il medio evo, 106, 2, 2004, p. 1-69.

${ }^{29}$ G. Tabacco, I liberi del re nell'Italia carolingia e postcarolingia, Spolète, 1966, p. 133-134 ; G. Musina, Le campagne di Piacenza tra VII e IX secolo : insediamenti e comunità, thèse de l'Université de Bologne, 2012, p. 106.

${ }^{30}$ Frédégaire, IV, 45 et Cont. 37-38.
} 
En 756, Aistulf dut ainsi livrer à Pépin le Bref le tiers du trésor du palais de Pavie ${ }^{31}$. Ajoutons le grignotage des curtes royales du fait des dons consentis par les gastalds ou les actores de manière autonome et frauduleuse : une dérive contre laquelle Liutprand s'élève en 724 , avant de promulguer, en 733, une série de normes de comportement et de gestion à l'intention des actores, visant entre autres à sanctionner toute appropriation illicite, que ce soit de leur fait ou à cause de l'aliénation de biens publics par des servi, aldii ou dépendants du roi ${ }^{32}$.

Les entrées, elles, peuvent être alimentées : par le paiement des amendes en espèces ou bien en terres, solution obligée dès que le montant devient élevé ; par le butin aux dépens des terres byzantines et romaines et par le tribut occasionnel ou durable, comme celui payé par l'Empire jusqu'aux années 640 ou celui versé par les Slaves aux ducs de Frioul jusqu'au temps du roi Ratchis, ou encore comme celui qu'Aistulf, en 751, voulut imposer de manière annuelle aux Romains, sous forme d'une capitation d'un sou d'or ${ }^{33}$; par les confiscations (inpublicatio) pour insolvabilité ou pour infidélité au roi $^{34}$; par les biens des personnes dépourvues d'héritier ou de «parents proches $»^{35}$; par le passage de femmes libres sous la potestas publica à cause de leur union avec des servi ${ }^{36}$. Il peut aussi y avoir des retours dans le fisc de biens cédés de manière temporaire, comme cela semble être le cas pour le gastald Alahis, dont le précepte qui lui accordait des salines est dit, dans l'inventaire, capillatus, c'est-à-dire «cassé » par une incision de la pièce de parchemin : peut-être parce que l'affectation de ce revenu à son profit n'avait plus de raison d'être après sa sortie de charge ${ }^{37}$.

Quoi qu'il en soit, il est impossible de savoir si les deux mouvements se compensaient. La légalisation des donations pieuses en $713^{38}$ a certainement détourné vers l'Église des biens qui auparavant seraient passés mécaniquement sous le contrôle du roi. Mais nous n'avons pas de moyen de juger de l'impact de ce manque à gagner. Toute approche strictement comptable des sorties du fisc, qui voudrait se fonder sur le nombre de curtes ou estimer des superficies, serait par ailleurs bien trop superficielle, en l'absence d'étude au cas par cas sur l'importance économique et/ou symbolique de chacun des biens cédés. Des progrès viendront de l'enquête en cours sur la Toscane ${ }^{39}$. Tout au plus peut-on relever, dès l'époque lombarde, que les biens provenant de confiscations sont réinjectés assez vite dans des donations, non à des individus mais à des monastères, comme le montrent les préceptes à destination de Farfa et de S. Salvatore de Brescia. Cela permet de ne pas entamer le capital « historique » de terres publiques tout en chargeant peut-être ces biens d'une valeur particulière : comme s'il y avait un double circuit de donations, l'un rapide, par lequel est redistribué ce qui arrive au roi de manière occasionnelle

\footnotetext{
${ }^{31}$ Frédégaire, Cont. 38. M. Hardt, Gold und Herrschaft. Die Schätze europäischer Könige und Fürsten im ersten Jahrtausend, Berlin, 2004, p. 40.

${ }^{32}$ Liutprand, 59 : si quis gastaldius vel actor curtem regiam habens ad gobernandum ex ipsa curte sine iussionem regis casa tributaria vel terram, silvam, vitis vel prata ausus fuerit donare... ; Notitia de actoribus regis. Voir C. Storti, «Le dimensione giuriduche della curtis regia longobarda », in Le corti nell'alto medioevo, Spolète, 2015 (Setimane di studio della Fondazione CISAM, 62), p. 429-472 : p. 443-444, 464-472 ; T. Lazzari, « La tutela del patrimonio fiscale $»$.

${ }^{33}$ Frédégaire, IV, 69 et Cont. 36 ; Paul Diacre, Historia Langobardorum IV, 38, MGH SSrL, p. 132 ; L. Duchesne, Le Liber pontificalis, I, Rome, 1886, p. 441. Gasparri, «Il regno longobardo », p. 38-40.

${ }^{34}$ Codice diplomatico longobardo, III, $\mathrm{n}^{\text {os }} 36,43,44$

35 Rothari 158-160, 163, 223, 231; Liutprand 17-18, 32, 34, 77 ; Benoît de Saint-André, Chronicon, éd. G. Zucchetti, Rome, 1920 (Fonti per la storia d'Italia, 55), p. 65 : Est autem constitutus in legibus Langobardorum, ut Langobardus qui sine heredes mortuus fuerit, curtis regia succedat. Voir aussi, dans le Midi, ce que dit le chroniqueur de Salerne à propos de Gumeltruda, femme du prince Adémar (853-861), qui se fait systématiquement donner par son époux, par voie de diplômes, les biens meubles et immeubles des défunts privés d'héritiers, passés dans le domaine princier sicuti mos est : Chronicon Salernitanum, éd. U. Westerbergh, Stockholm, 1956, p. 101.

${ }^{36}$ Codice diplomatico longobardo, III, $\mathrm{n}^{\text {os }}$ 18, 43, 44 Rothari 221, Liutprand 24 ; voir aussi Adelchis 1.

${ }^{37}$ Codice diplomatico longobardo, III, p. $268 \mathrm{n}^{\circ} 6$ : Et precepto capillato, que Liutprand emiserat in Alahis de salinas.

${ }^{38}$ Liutprand 6.

${ }^{39}$ Voir la contribution de Giovanna Bianchi et Federico Cantini dans le présent volume.
} 
et parfois controversée ; l'autre plus lent, qui affecte le cœur des possessions. Le phénomène, qui a caractérisé aussi la période carolingienne, est comparable à la mobilité observée pour les éléments périphériques des patrimoines privés, comme ceux qui ont pu constituer la «Mogengabe », plus facilement mise sur le marché de la terre que le reste des biens familiaux ${ }^{40}$.

Il faut encore mettre au compte des souverains lombards un changement notable dans l'affectation de larges étendues foncières dépendant du fisc, par le biais du transfert à des établissements monastiques. Ce mouvement, sur lequel a récemment insisté Tiziana Lazzari ${ }^{41}$, a duré le temps d'une génération, débutant au milieu du vIII ${ }^{\mathrm{e}}$ siècle avec Ratchis et se poursuivant sous Aistulf et Didier, pour culminer avec la fondation de S. Salvatore de Brescia. Il faut toutefois distinguer entre les monastères anciens (Farfa, S. Vincenzo al Volturno) et les fondations récentes comme Nonantola, S. Salvatore al Monte Amiata, Leno et Brescia. Farfa et S. Vincenzo al Volturno reçoivent des gualdi, sur lesquels leur gestion vient se surimposer à celle des communautés qui en avaient jusque-là la jouissance, ce qui est source de conflits récurrents et durables. Pour les autres, il fallait constituer une dotation, dans la plupart des cas ex nihilo: c'est-à-dire autant de soustrait aux appétits des actores royaux dont Liutprand stigmatisait la corruption $^{42}$.

Ce phénomène peut être apprécié selon plusieurs critères. Celui de la « mise en réserve » du fisc par la soustraction à la gestion ordinaire ne me convainc qu'à moitié, car les aliénations consenties furent définitives et ne paraissent pas avoir été compensées, pour autant qu'on le sache, par une augmentation particulière des contributions des monastères destinataires en faveur de la royauté. Tout au plus peut-on dire qu'avait été trouvé là un moyen radical de mettre fin à l'érosion graduelle dénoncée par le législateur, au profit de Dieu plutôt qu'à des individus. Il faut replacer ces donations dans la chronologie générale des fondations monastiques, qui battent leur plein au VIII ${ }^{\mathrm{e}}$ siècle et pour lesquelles les souverains lombards désormais pleinement catholiques ne pouvaient être en reste par rapport aux initiatives plus modestes et plus diffuses. Le critère du contrôle trop facilement qualifié de «stratégique » du territoire ne me paraît pas non plus pleinement satisfaisant - la proximité entre Leno et de S. Salvatore de Brescia suffit pour en douter -, et ne peut guère s'appliquer qu'au Monte Amiata, créé avec le soutien d'Aistolf puis promu par Adelchis et dans lequel il est difficile de ne pas reconnaître un instrument au service de l'organisation du territoire, à proximité immédiate de la Tuscie romaine et en lien direct avec les voies de pèlerinage ${ }^{43}$. La fondation majeure, S. Salvatore de Brescia, assortie d'un mausolée pour Didier et Ansa, est en revanche un cas différent. Elle répond d'une part à un souci de promotion dynastique sur les lieux mêmes dont Didier était originaire : l'initiative est celle du couple et la dotation première naît de leur propria facultas ${ }^{44}$. D'autre part, elle assure à la reine et à sa fille Anselperga, qui fut la première abbesse, une indépendance économique et une capacité d'action inédites dans le royaume : cette position renforce la position institutionnelle de la souveraine, ce dont ont dû tenir compte ceux qui ont occupé le trône par la suite. C'est en ce sens que la réserve patrimoniale constituée en partie sur le fisc, fossilisé dans sa nouvelle vocation, sert positivement la royauté, plus que ne le font les autres donations.

\footnotetext{
${ }^{40}$ L. Feller, " "Morgengabe", dot, tertia : rapport introductif », in in Id., L. Feller et R. Le Jan (éd.), Dots et douaires dans le haut Moyen Âge, Rome, 2002 (Collection de l'École française de Rome, 295), p. 1-25 : p. 21 ; F. Bougard «Dot et douaire en Italie centro-septentrionale, $\mathrm{VIII}^{\mathrm{e}}-\mathrm{XI}^{\mathrm{e}}$ siècle : un parcours documentaire », ibid., p. 58-95: p. 68-69.

${ }^{41}$ Lazzari, « La tutela del patrimonio fiscale », p. [10]-[12].

${ }^{42}$ Supra, note 19.

${ }^{43}$ W. Kurze, « Monasterium Erfonis. I primi tre secoli di storia del monastero e la loro tradizione documentaria » [1986], in Id., Monasteri e nobiltà nel Senese e nella Toscana medievale. Studi diplomatici, archeologici, giuridici e sociali, Sienne, 1989, p. 357-374 : p. 358-360.

${ }^{44}$ Codice diplomatico longobardo, III, $\mathrm{n}^{\mathrm{o}} 31$.
} 
À partir de 774, et à mesure du remplacement des ducs et des gastalds à pouvoir ducal par des comtes francs, le changement de domination politique a aligné les pratiques en vigueur dans le regnum sur celles du nord des Alpes. Cependant, l'attitude des souverains carolingiens face à ce nouveau réservoir de biens publics a changé au cours du temps. Il a fallu plus d'une génération pour passer d'une prise en main marquée par la sévérité, à un régime plus ordinaire.

Au lendemain de la conquête, la priorité était d'arrimer le nouveau territoire à la Francie. D'où les aliénations de la Valcamonica, de la Valteline et de Bardonecchia en faveur de SaintMartin de Tours, Saint-Denis et Saint-Pierre de Novalesa ${ }^{45}$. Des considérations d'ordre véritablement stratégique étaient assurément au premier plan, pour le contrôle de ces points de passage et des cluses qui les défendaient. Cependant, ces dons pouvaient être aussi intéressants pour ce qu'ils offraient en ressources d'huile d'olive (pour le luminaire) dans la région des lacs préalpins et jusqu'en Valteline, ainsi qu'en minerai - bien que l'exploitation des filons argentifères bergamasques ne soit pas attestée avant $1 \mathrm{e} \mathrm{XI}^{\mathrm{e}}$ siècle ${ }^{46}$. Saint-Denis obtint de Lothaire $\mathrm{I}^{\mathrm{er}}$ un droit de marché sur le lac de Côme en 833, avec les revenus fiscaux afférents, et l'on peut penser que l'attachement durable de Saint-Martin de Tours à ses possessions italiennes n'est pas étranger à de telles préoccupations d'ordre économique ${ }^{47}$. Une formulation en partie commune aux diplômes accordés à Tours et à la Novalesa doit surtout être relevée, qui souligne que les biens proviennent « du public et du palais (lombard) », sicut in publico et ad palatium visum est pertinuisse $e^{48}$, et sont «désormais tombés dans notre fisc », et inantea intro fisco nostro ceciderit. On ne saurait mieux exprimer le transfert de domination : les biens du régime précédent sont mobilisés au profit des Francs. Le regnum Langobardorum est certes maintenu dans son identité politique, mais n'a plus d'autonomie quant à l'usage de ses propres biens. Le registre est celui de la prédation, qui veut aussi que le trésor du palais de Didier, le roi vaincu, passe dans les caisses de Charlemagne, de même que celui d'Arechis de Bénévent en 787, plutôt qu'il ne contribue à soutenir la royauté en Italie, fût-elle-même déléguée à Pépin ${ }^{49}$.

Les premiers temps ont été marqués par la volonté d'affirmer avec force les droits du nouveau régime. Les diplômes de Charlemagne manifestent le souci d'afficher une rupture, que l'on dit fondée en droit. En 797 furent ainsi donnés à S. Silvestro de Nonantola des biens que le roi Liutprand avait lui-même cédés en emphytéose au Grec Grégoire, son iocator, puis qui étaient passés sous le dominium du roi franc: un passage iure legitimo, prend-on soin de préciser $^{50}$. L'exemple le plus significatif est toutefois fourni par la manière dont fut parfois justifié le maintien des largesses des souverains précédents. L'abbaye S. Maria in Sylvis, en Frioul, avait obtenu du roi Adelchis, ex fisco nostro, dit un diplôme de Charlemagne de 781, ce

\footnotetext{
${ }^{45}$ MGH DD Kar. I 81, 94 ; DD Lo I 92.

${ }^{46}$ Sur l'usage liturgique de l'huile d'olive et sur l'oléiculture dans la région, M. Montanari, L'alimentazione contadina nell'alto medioevo, Naples, 1979, p. 396-404 ; G. M. Varanini et A. Brugnoli, «Olivi e olio nel patrimonio della famiglia di Totone di Campione », in S. Gasparri et C. La Rocca (éd.), Carte di famiglia. Strategie, rappresentazione e memoria del gruppo familiare di Totone di Campione (721-877), Rome, 2005, p. 141-156. A. Stoclet, Autour de Fulrad de Saint-Denis (v. 710-784), Paris, 1993, p. 434, insiste sur la «politique minière » des Carolingiens, qui reste toutefois à démontrer dans le cas de l'argent bergamasque.

${ }^{47}$ Saint-Denis : MGH DD Lo. I 13 ; voir aussi DD 100, de 848, restitution à Saint-Denis de la Valteline, qui lui avait été retirée durant le conflit entre Louis le Pieux et Lothaire. — Saint-Martin : P. Baccou, «Sur un acte prétendu faux de Bérenger $\mathrm{I}^{\mathrm{er}}$, roi d'Italie, pour Saint-Martin de Tours », Mélanges de l'École française de Rome. Moyen Âge, 115, 2003, p. 711-725 ; MGH DD O. II 233, DD O. III 289.

${ }^{48}$ La formulation in publico et ad palatium n'est pas redondante, mais reproduit une distinction déjà signalée : supra, texte correspondant à la note 20 .

${ }^{49}$ Didier : Annales regni Francorum, éd. F. Kurze, MGH SS rer. Germ. 6, a. 774, p. 38. — Arechis : Erchempert, Historia Langobardorum Beneventanorum, 2, MGH SSrL, p. 235. Hardt, Gold und Herrschaft, p. 41.

${ }^{50}$ MGH DD Kar. I 183.
} 
que le vicus de Sacco avait coutume de verser à «notre » curtis ducale de Trévise, à savoir cent muids de seigle et cinquante porcs. Adelchis avait par ailleurs confirmé des biens sis dans le territoire de «notre cité » de Cividale, ainsi qu'un échange avec un gastald, aux termes duquel l'abbaye était entrée en possession de terres de curte nostra. Or tout cela est illégal, déclare Charlemagne après en avoir discuté avec ses fideles et ses proceres. "C'est pourquoi », poursuit le diplôme de manière apparemment paradoxale, l'ensemble des biens en question est donné au monastère ${ }^{51}$. L'acte, transmis en copie du Xv $\mathrm{V}^{\mathrm{e}}$ siècle, peut avoir été simplifié dans certaines de ses formulations, mais n'est pas suspect. Le point important est que le monastère dispose de ces biens non pas à cause de la confirmation d'un prélèvement sur le fisc fait par le dernier roi lombard, mais ex nostra largitate : manière de faire table rase du passé. Mais pourquoi procéder ainsi ? On peut supposer que la donation/confirmation d'Adelchis fut contestée par les nouveaux responsables du fisc local. L'affaire fut portée devant Charlemagne, qui a tranché. Rien n'a changé pour Sesto, mais il devient manifeste à tous - le diplôme est adressé à l'ensemble des autorités - que, en matière de concession de biens publics, rien n'est acquis, spécialement pour ce qui fut aliéné dans les temps précédant immédiatement la conquête.

Pour les biens qui avaient été cédés par les ducs, les mesures furent plus strictes. Charlemagne ordonna de les réintégrer dans le domaine royal. Au cours d'une séance de justice tenue à Norcia en 821 devant Adalard de Corbie, le duc franc de Spolète Winigis, qui avait succédé au Lombard Hildeprand en 787, expliqua ainsi avoir rappelé ad regiam partem, comme cela lui avait été demandé, l'ensemble de ce que les uns et les autres avaient pu obtenir per precepta ducum ${ }^{52}$.

D'autres procès témoignent du fait que, pendant plusieurs décennies, qui correspondent à peu près à celles des règnes de Charlemagne et de Pépin, et parfois plus tard, les nouvelles autorités ont cherché à récupérer ce qui pouvait l'être et à remettre en cause les concessions des rois lombards ${ }^{53}$. Les notices conservées ne renvoient l'écho que des affaires gagnées par les anciens bénéficiaires, des monastères puissants, et il s'agit selon toute vraisemblance de l'arbre qui cache la forêt des retours dans le publicum. S. Zeno de Vérone fut ainsi expulsé per fortiam par le premier comte franc du lieu, Vulvanus, de biens qu'il tenait en vertu d'un précepte de Didier. La position du monastère était d'autant plus fragile que le document avait péri lors de l'incendie de la domus sancti Zenonis. Il ne fut rétabli dans ses droits qu'en 806, à l'occasion d'un plaid qui l'opposa au gastald représentant la causa regis sur ordre du comte Hadumar ${ }^{54}$. En 820 encore, le comte de Vérone fut cité en justice par l'abbé de S. Silvestro de Nonantola pour avoir usurpé ce que tenait le monastère dans la forêt d'Ostiglia et qui provenait du duc Anselme, fondateur de l'établissement ; celui l'avait acquis du roi, probablement Aistulf, dont il était le beau-frère ${ }^{55}$.

\footnotetext{
${ }^{51}$ MGH DD Kar. I 134.

${ }^{52}$ I Placiti del «Regnum Italiae », éd. C. Manaresi, Rome, 1955-1960, 3 t. en 5 vol. (Fonti per la storia d'Italia, 92, 96) : I, no 32, p. 102 : ipsas res ad regiam parem recolligere feci, sicut alias res aliorum hominum, quas per precept ducum habuerunt, et sicut commendatum michi fuit a domno Karolo imperatore.

${ }^{53}$ Andrea Castagnetti avait déjà insisté sur ce durcissement : «Immigrati nordici, potere politico e rapporti con la società longobarda », in S. de Rachewiltz et J. Riedmann (éd.), Kommunikation und Mobilität im Mittelalter. Begegnungen zwischen dem Süden und der Mitte Europas (11.-14. Jahrhundert), Sigmaringen, 1995, p. 27-60 : p. 37.

${ }^{54}$ Placiti, I, no 18. Sur Vulvanus/Vulfuinus, E. Hlawitschka, Franken, Alemannen, Bayern und Burgunder in Oberitalien (774-962). Zum Verständnis der fränkische Königsherrschaft in Italien, Freiburg im Br., 1960, p. 292293 ; A. Zettler, « Die karolingischen Grafen von Verona : Überlegungen und Annäherungsversuche », in H. Krieg et A. Zettler (éd.), In frumento et vino optima : Festschrift für Thomas Zotz zu seinem 60. Geburtstag, Ostfildern, 2004, p. 89-114 : p. 97-99.

${ }^{55}$ Placiti, I, no 31 ; A. Castagnetti et A. Ciaralli, Falsari a Nonantola.I placiti di Ostiglia (820-827) e le donazioni di Nogara (910-911), Spolète, 2011, p. 265-273.
} 
Le zèle de l'autorité fiscale ne s'est au reste pas limité à ce qui avait été cédé avant 774 . L'impression prévaut que tout détenteur de biens provenant du fisc est susceptible d'une contestation en justice ou d'une récupération de fait. À Crémone, au temps de Pépin, le baiulus Rotechildus annule toutes les donations à l'église locale, y compris ce qu'avait accordé un précepte de Charlemagne ${ }^{56}$. En 818 , le monastère de Nonantola est aux prises avec la curtis royale de Mantoue, qui lui conteste divers droits d'usage (pâture, chasse, pêche) et revenus pourtant détenus en vertu d'un diplôme d'Aistulf régulièrement confirmé depuis par Didier, Charlemagne puis Louis le Pieux ${ }^{57}$. De même, l'affectation de biens pour constituer des bénéfices en rémunération de service public se fait volontiers à l'aveugle ou par la force sciemment ou par défaut de mémoire de l'institution ? En 811, Farfa poursuit ainsi en justice un gastald au sujet de terres et de vignes appartenant à l'abbaye mais qui ont été attribués en bénéfice pro publica causa à son adversaire ${ }^{58}$. Environ une dizaine d'années plus tôt, le baiulus Rotechildus, encore lui, avait profité du fait qu'il avait envoyé en exil l'abbé de S. Bartolomeo de Pistoia pour confier le monastère en bénéfice au Bavarois Nibelung ${ }^{59}$.

L'attention sourcilleuse envers les droits du publicum et la recherche systématique de tout ce qui s'y rattache, avec une volonté marquée à remettre en cause ce qui avait pu être concédé au titre des rois ou des ducs lombards, s'accompagne d'une tendance non moins nette à un alourdissement des prélèvements. Au moins dans les premières décennies qui ont suivi la conquête, le régime est décidément plus exigeant qu'auparavant, d'où des protestations et des conflits. On reproche d'une part d'augmenter ce qui existe déjà (ce sont les superpostae), d'autre part de prétendre lever des taxes nouvelles. Les marchands de Comacchio se plaignent en 781 d'une surtaxe de $50 \%$, exigée en particulier à Mantoue ${ }^{60}$. En revenant d'exil, l'abbé de S. Bartolomeo de Pistoia déjà cité put certes recouvrer sa charge, tandis que Nibelung fut écarté. Mais ce dernier ne laissa pas moins une mala consuetudo, sous forme d'obligations inédites auxquelles il ne fut pas facile de se soustraire: le service militaire par l'abbé alors que le fondateur du monastère avait laissé des héritiers pour répondre à ce devoir; des paratas et coniectas pour les missi du roi ; un versement (datio) semble-t-il annuel au palais ${ }^{61}$. Voici encore, en 835, le clergé de Crémone, dont l'on prétend «injustement » qu'il fournisse des palefrois et des charrois auprès de la camera royale, c'est-à-dire à la curtis de Sospiro ${ }^{62}$.

Le plus notable est ce qui ressort de l'enquête menée vers 804 à Risano/Rižana sur le régime mis en place par le duc Jean, représentant carolingien, en Istrie ${ }^{63}$. La région, auparavant sous domination byzantine, n'est certes pas représentative du reste du royaume et son caractère excentré rend plus facile la tentation de l'extraordinaire et de l'arbitraire, ce qui n'est pas sans rappeler ce dont se plaignait le patriarche de Grado face aux collectae abusives et répétées qu'infligeait le pouvoir lombard sur les dépendants de son église ${ }^{64}$. Quoi qu'il en soit le contraste entre les pratiques de la nouvelle administration et les usages antérieurs en dit long sur les crispations qu'a pu susciter le changement des pouvoirs. Le plaid offre d'abord l'occasion de constater le gouffre qui sépare le régime fiscal de Byzance et celui de l'Occident. « Du temps des Grecs », chaque ville devait payer un impôt (iustitiae) annuel, en monnaie, pour

\footnotetext{
${ }^{56}$ Placiti, I, n 119 et Inquisizioni, $\mathrm{n}^{\mathrm{o}}$ VII.

${ }^{57}$ Placiti, I, no 30 (=ChLA, 2 ${ }^{\mathrm{e}}$ s., LXXXVIII, Dietikon-Zurich, 2008, $\mathrm{n}^{\mathrm{o}} 31$ ).

${ }^{58}$ Placiti, $\mathrm{I}, \mathrm{n}^{\circ} 24$.

${ }^{59}$ Placiti, I, no 25 ; M. Stoffella, «Le relazioni tra Baviera e Toscana tra VIII e IX secolo", Mélanges de l'École française de Rome. Moyen Âge, 120, 2008, p. 73-87: p. 76-77.

${ }^{60}$ MGH DD Kar. I 132 : les nouveaux maîtres exigent des versements en muids d'une capacité de quarante-cinq livres, contre trente précédemment.

${ }^{61}$ Placiti, I, n ${ }^{\circ} 25$.

${ }^{62}$ MGH DD Lo I 25.

${ }^{63}$ Placiti, I, n ${ }^{\circ} 17$.

${ }^{64}$ MGH Epp. III, p. 712-713 ; S. Gasparri, « Il regno longobardo », p. 37 ; Id., « Le basi economiche », p. 82.
} 
un total de 344 mancosi (ici, clairement, des nomismata ${ }^{65}$ ), à destination du palais impérial. Or le duc Jean, lui, continue de prélever, mais pour son usage personnel, s'indigne-t-on, alors qu'il dispose de ressources, de colons, de pêcheries, au titre du fisc ${ }^{66}$. En d'autres termes, il confond la régie des recettes et n'a aucune notion de ce à quoi doit servir un impôt direct, notamment dans sa redistribution. Les liquidités dont il dispose ne sont pas pensées comme l'élément d'un budget, qui pourrait inclure par exemple le financement militaire, selon les règles que les maîtres de Constantinople rappelèrent encore à Liudprand en 968, menaçant de lancer contre Otton toutes les nations grâce à l'argent qui fondait la puissance de l'empire d'Orient ${ }^{67}$.

Il serait trop long d'énumérer tout ce que les habitants de l'Istrie considèrent comme des abus par rapport à ce qui leur était demandé auparavant ${ }^{68}$. Il en ressort que le duc met le pays en coupe réglée au profit de ses fils, de ses filles et de son gendre. Mais tout n'est pas que prévarication, car il y aussi une différence d'appréciation de ce que devraient être les droits de chacun. À propos des pâturages, pour lesquels, comme sur tant d'autres sujets, sont imposées des angarias et superpostas au point que les gens d'Istrie sont devenus la risée de leurs voisins de Vénétie, de Dalmatie et même des Grecs, la seule défense est qu'il « croyait » que ces bienslà revenaient au publicum. N'y voyons pas malignité, mais plutôt la difficulté à penser certains espaces ruraux comme les pacages et les forêts autrement qu'en relation directe avec le fisc ${ }^{69}$.

L'enquête de 804 montre aussi le poids nouveau des «dons » au palais. Alors que pour les sujets du basileus, le don est une occasion de contact régulière et ritualisée avec l'empereur, le duc, lui, rassemble le tout et le porte lui-même en Francie, court-circuitant ainsi la relation directe entre la population et le souverain. Les dona annualia, forme d'imposition typiquement carolingienne mais dont nous avons vu qu'elles faisaient déjà partie d'une pratique consolidée à l'époque lombarde, sont à mi-chemin entre la contribution récognitive et la fiscalité ${ }^{70}$. Ils sont récognitifs d'une sujétion à l'Empire, par exemple, quand ils sont envoyés depuis Rome à Pavie, selon le témoignage - que l'on peut estimer sujet à caution - du Libellus de imperatoria potestate in urbe Roma : dix livres d'or et cent d'argent, dix pallia optima, auxquels s'ajoutent d'éventuels dons privés ${ }^{71}$. Récognitifs, aussi, pour un monastère comme celui de S. Andrea de Florence, qui avait coutume au milieu du $\mathrm{IX}^{\mathrm{e}}$ siècle de faire parvenir au palais, chaque année, vestitum unum bonum caprenum, avant que Louis II n'en fît bénéficier l'évêque du lieu ${ }^{72}$. Mais la démultiplication de ces dons dans l'ensemble du royaume, leur caractère systématique à tous les échelons ainsi que le langage employé à leur propos (persolvere) les placent décidément sur le terrain de la fiscalité, comme au nord des Alpes ${ }^{73}$ : celle dont s'acquittent les responsables

\footnotetext{
${ }^{65}$ V. Prigent, « Le mythe du mancus et les origines de l'économie européenne », Revue numismatique, 6 $6^{\mathrm{e}}$ s., 171, 2014, p. 701-728.

${ }^{66}$ ad suum opus istos solidos habuit et non dixit quod iustitia pallatii fuisset.

${ }^{67}$ Liudprand de Crémone, Legatio 53 : pecunia, qua pollemus, omnes nationes super ipsum invitabimus...

${ }^{68}$ Le texte a fait l'objet de nombreuses études, spéc. S. Esders, « Regionale Selbstbehauptung zwischen Byzanz und dem Frankenreich. Die inquisitio der Rechtsgewohnheiten Istiens durch die Sendboten Karls des Großen und Pippins von Italien», dans Id. et Th. Scharff (éd.), Eid und Wahrheitssuche. Studien zu rechtlichen Befragungsparktiken in Mittelalter und früher Neuzeit, Francfort, 1999, p. 49-112 ; voir en dernier lieu les contributions réunies dans Acta Histriae, 13, 1, 2005.Traduction française par P. Depreux, Les sociétés occidentales du milieu du VI è la fin du IX siècle, Rennes, 2002, p. 293-299.

${ }^{69}$ La même remarque vaut pour le litige entre le comte de Vérone et le monastère de Nonantola à propos de la forêt d'Ostiglia (supra, texte correspondant à la note 55). Voir A. Castagnetti, « Il conte Anselmo I : l'invenzione di un conte carolingio », Studi storici Luigi Simeoni, 56, 2006, p. 9-60 : p. 33-34.

${ }^{70}$ Hincmar, De ordine palatii 22 et 29, éd. Th. Gross et R. Schieffer, MGH Fontes iuris 3, p. 72 et 84 ; supra, texte correspondant à la note 24.

${ }^{71}$ Libellus de imperatoria potestate in urbe Roma, éd. G. Zucchetti, Rome, 1920 (Fonti per la storia d'Italia, 55), p. 195.

72 R. Piattoli, Le carte della canonica della cattedrale di Firenze (723-1149), Rome, 1938 (Regesta chartarum Italiae, 23), n 2. Voir aussi la datio due par S. Bartolomeo de Pistoia citée plus haut.

${ }^{73}$ Hincmar établit l'équivalence entre les annua dona et les regi ac rei publicae vectigalia : De ordine palatii 22 MGH Fontes iuris 3, p. 72, note 165.
} 
régionaux quand ils se présentent au plaid général et dont on trouve un écho, à un échelon inférieur, dans la mention d'annuales donationes collectées par les exactores, au détour d'un diplôme accordé en 883 à l'église de Bergame ${ }^{74}$. N'oublions pas non plus ce que les uns et les autres ont pu apporter au palais à l'occasion de tel ou tel événement particulier, par exemple le baptême de la fille de Lothaire, pour lequel l'archevêque de Ravenne fit des dépenses somptuaires aux dépens du trésor de son église, à la fois pour les exenia dus à l'empereur et pour l'acquisition de vêtements de cérémonie fabriqués au palais ou dont celui-ci avait le monopole de la vente ${ }^{75}$.

Les prestations dont l'église de Novare fut exemptée en 854 étaient plus probablement étalées durant l'année. Était concerné tout ce que le «droit du fisc » avait pu exiger sur ses biens, à savoir annona, vinum, caseum, pulli, ova, castaneae fructusque messis atque quae lentibus gignitur plustacia, calcem, venationes vel ceterorum alia ${ }^{76}$. Où l'on trouve non seulement des denrées alimentaires, dont la liste fait irrésistiblement à celles que l'on peut lire au fil du capitulaire de villis et qui pourrait être complétée au gré de mentions éparses jusqu'à la fin $\mathrm{du} \mathrm{X}^{\mathrm{e}}$ siècle, mais aussi la fourniture de la chaux, qui faisait partie des obligations contestées par les habitants de l'Istrie en $804^{77}$. Certaines des terres prélevées sur le temporel monastique pour être affectées en bénéfice semblent en revanche avoir été soumises à une contribution monétaire, comme cela ressort du polyptyque de Bobbio dressé dans les dernières années du IX ${ }^{\mathrm{e}}$ siècle $^{78}$.

Est-ce à dire qu'en dehors de ces versements exigés au fil de l'année par les responsables locaux ou par les missi, ou bien versés une fois l'an par les collectivités (des villes, des monastères), les uns en monnaie, les autres en produits alimentaires, en objets ou en services, les Carolingiens ne savent pas lever l'impôt direct ? Ce n'est pas tout à fait le cas. Il leur arrive d'abord de faire appel à des contributions extraordinaires, comme cette collatio pecuniae imposée par Lothaire à l'ensemble de l'Empire pour financer la fortification de la cité Léonine, au lendemain du pillage de Saint-Pierre par les Sarrasins ${ }^{79}$. En Italie comme en Francie, l'impôt direct réapparaît aussi sous la forme d'un prélèvement sur la production, dicté au même moment de part et d'autre des Alpes et dont la progression suit semble-t-il le même rythme ici et là dans le courant $\mathrm{du} \mathrm{IX}^{\mathrm{e}}$ siècle : c'est la dîme, dont il faut souligner qu'elle est versée à une institution, l'Église, tout aussi « publique » que l'État, avec lequel l'historiographie ne cesse au reste de l'identifier. En schématisant, on pourrait dire qu'a été mis en place un transfert de ressources, d'une caisse vers une autre. Ce n'est pas la capacité technique du prélèvement fiscal qui est en cause, c'est le fait que ses acteurs changent, et avec eux les secteurs de la redistribution. Il est certes possible que certains aient bénéficié des deux systèmes, puisque la dîme ne finance certes ni l'armée ni « l'administration », mais a pu être affectée, en partie, en ville, à ce que l'on peut assimiler au ravitaillement ou à la défense. De même, les dîmes perçues par certains monastères ont pu servir à l'entretien des abbés laïcs, là où il y en eut. Mais en remettant à l'ordre du jour

\footnotetext{
${ }^{74}$ MGH DD Kar. III 89, p. 147 1. 8.

${ }^{75}$ Agnellus, Liber pontificalis ecclesiae Ravennatis 171 : post omnia exenia augustali tributa, emit ex palatio eius... vestimenta baptismalia...

${ }^{76}$ MGH DD L II 14 ; Brühl, Fodrum, p. 448-449.

${ }^{77}$ Sur la fourniture de la chaux, qui passe par la construction de fours, également attestée à Bénévent ; F. Bougard et É. Hubert, «La fabrication de la chaux en Italie centrale au Moyen Âge », in Liber amicorum. Études offertes à Pierre Bougard, Arras, 1987, p. 57-64 : p. 61 ; J.-M. Martin, Guerre, accords et frontières en Italie méridionale pendant le haut Moyen Âge. Pacta de Liburia, Divisio principatus Beneventani et autres actes, Rome, 2005, p. 194. ${ }^{78}$ Inventari altomedievali di terre, coloni e redditi, Rome, 1979 (Fonti per la storia d'Italia, 104), p. 169-170, 172174 (fisco solidum I, fisco denarios XIII, fisco solidos II etc.) ; voir aussi p. 133 et 154 ; pour la datation du document, Bougard, La justice, p. 383.

${ }^{79}$ MGH Capit. II, no 203 , c. 7-8, p. 66-67.
} 
une forme d'impôt, on ne songeait pas précisément à en affecter le produit à la manière antique. Il s'agit bien d'un choix, non d'une incompétence.

L'autre élément de ce choix est de miser au quotidien sur l'impôt indirect, sur lequel les sources sont prolixes et dont Carlrichard Brühl a donné une présentation fournie. L'impôt indirect figure dans la formule d'immunité cum fredis concessis (c'est-à-dire les frais de justice) dont bénéficient sous Charlemagne plusieurs monastères et églises épiscopales, dès $773^{80}$. Mais il est aussi volontiers détaillé au détour de tel ou tel diplôme. Ainsi en 822, dans le tout premier diplôme de Lothaire, qui exempte un bateau de commerce de l'abbaye de Farfa des taxes ordinaires, où le vocable générique «tonlieu » ${ }^{81}$ est détaillé pour tous les aspects relatifs à la circulation marchande, voire au-delà : ripaticum, portaticum, pontaticum, salutaticum, cespitaticum, cenaticum, pastionem, laudaticum, travaticum, pulveraticum, occursus, census, redibitio. Autant de taxes dont la signification n'est pas toujours limpide, mais dont on perçoit aisément qu'elles se répartissent entre les opérations liées à la circulation, à l'accostage et aux ruptures de charge d'une part, et ce que perçoivent les différents représentants de la chose publique au fil du trajet. En 820, un autre diplôme pour Farfa concerne cette fois l'exemption de ce qui est dû pour l'usage des pâturages publics dans le duché de Spolète : y figurent cette fois daticum, aescaticum, herbaticum, glandaticum, c'est-à-dire une liste déjà présente dans un diplôme de Didier mais augmentée de la glandée ; en 840, un acte de Lothaire y ajoute le castaldaticum, c'est-à-dire les droits perçus par le gastald ${ }^{82}$.

Il serait vain de penser dresser un panorama non seulement complet mais réaliste de la taxation à partir de telles énumérations, si riches soient-elles. Si la liste des produits dus par l'église de Novare peut être considérée comme fiable, émanant selon toute vraisemblance du destinataire même de l'acte, celle du diplôme accordé à Farfa en 822 n'est que le calque d'une formule impériale mise au point pour la Gaule ${ }^{83}$. Par ailleurs, le souci d'exhaustivité que l'on devine n'a probablement d'égal que l'inventivité des représentants du fisc. Il est donc aussi efficace, et toujours avisé, d'ajouter une formulation susceptible de tout rassembler, y compris ce à quoi l'on n'avait pas pensé : « et tout ce que le fisc avait pu espérer », précise le diplôme de 822 pour Farfa déjà cité ${ }^{84}$, selon une expression en vigueur de part et d'autre des Alpes et qui, souvent, dispense même d'en dire davantage. Elle trouve encore un écho en 896 dans un diplôme d'Arnulf pour S. Sisto de Plaisance : après la mention de taxes génériques (tonlieu, ripaticum, portaticum) susceptibles d'être perçues par les exactores de la pars publica dans les mansiones du monastère, les marchés publics, les ports ou autres lieux, vient cette précision que l'exemption concerne aussi bien tout ce qui, en matière d'exaction publique, "peut être dit ou nommé ${ }^{85}$. On n'est jamais trop prudent et il en va de la diversité des taxes comme des malédictions bibliques des clauses de sanction spirituelle, parfois décrites par le menu, parfois simplement réunies dans la formule «toutes les malédictions de l'Ancien et du Nouveau Testament » ou « les imprécations habituelles », ce qui est encore le meilleur moyen de ne pas en oublier ${ }^{86}$.

\footnotetext{
${ }^{80}$ MGH DD Kar. I 74 (Novalesa), 99 (Farfa), 133 (Reggio), 135 (S. Salvatore de Brescia), 147 (Modène), 157 (S. Vincenzo al Volturno); diplôme perdu pour Volterra (MGH DD L I 199 = Lechner 574). Sur les freda et leur répartition, F. L. Ganshof, «Charlemagne et les institutions de la monarchie franque », dans H. Beumann (éd.), Karl der Grosse. Lebenswerk und Nachleben, I, Düsseldorf, 1965, p. 394-419 : p. 379-380 ; J. Durliat, Les finances publiques de Dioclétien aux Carolingiens (284-899), Sigmaringen, 1990 (Beihefte der Francia, 21), p. 219.

${ }^{81}$ F. L. Ganshof, « À propos du tonlieu à l'époque carolingienne », dans La città nell'alto medioevo, Spolète, 1959 (Settimane du studio del CISAM, 6), p. 485-508; Durliat, Les finances publiques, p. 217.

${ }^{82}$ MGH DD L I 178, p. 443 ; Codice diplomatico longobardo, III, no 43, p. 250 ; MGH DD Lo. I 51, p. 152.

${ }^{83}$ MGH Formulae, p. 301.

${ }^{84}$ MGH DD Lo. I 1 : Et quicquid exinde fiscus sperare poterat aut ad ministros mundanae actionis pervenire...

${ }^{85}$ MGH DD Arn 141 : nihilque publice exactionis, quod dici aut nominari potest.

${ }^{86}$ F. Bougard, «Jugement divin, excommunication, anathème et malédiction : la sanction spirituelle dans les sources diplomatiques », in G. Bührer-Thierry et S. Gioanni (dir.), Exclure de la communauté chrétienne. Sens et
} 
L'existence même de ces prélèvements indirects est de nature à susciter le conflit, spécialement quand leur bénéficiaire n'est pas la pars publica et quand surgit une taxe considérée comme nouvelle aux yeux de qui doit la payer. C'était le cas en Istrie, nous l'avons vu. Il en va de même à Crémone, où l'affaire est au cœur du litige qui a durablement opposé l'évêque et les cives. Charlemagne avait concédé à l'Église de Crémone une curtis sur le Pô, le port de Vulpariolo et « les autres ports » entre la ville et l'embouchure de l'Adda. S'y ajoutaient un droit de mouture sur les moulins et un droit sur la circulation (transitoria) des bateaux ainsi que sur celle des marchands (milites) de Comacchio. Au milieu du $\mathrm{IX}^{\mathrm{e}}$ siècle, les habitants se plaignirent de ce que l'évêque les traitait comme les gens de Comacchio, en exigeant d'eux, qui plus est par la force, une série de taxes selon eux inédites (ripaticum, palifictura, pastum) au port de la cité. L'évêque, lui, se considérait dans son bon droit, estimant pouvoir prélever sur tout negotiator, quelle que fût son origine. Il obtint gain de cause, puis fit confirmer l'issue du plaid par Louis II $^{87}$. Plusieurs facteurs se mêlent pour expliquer ce litige. D'une part, le fait que les Crémonais étaient nouveaux dans l'armement de navires pour le transport du sel et autres denrées, alors que jusqu'il y a peu, ils avaient opéré sur ceux de Comacchio, payant les taxes de manière commune avec les représentants de l'emporium. D'autre part, l'église de Crémone n'avait été réintégrée dans ses prérogatives que peu d'années auparavant, après de nombreuses années durant lesquelles les revenus indirects avaient fait retour au fisc, sur l'initiative autoritaire du baiulus Rotechildus, déjà nommé ${ }^{88}$. Enfin, le prélat en charge, Benoît (843/851878/880), ancien chapelain de l'empereur, avait visiblement une interprétation du précepte de Charlemagne plus extensive que celle de son prédécesseur ${ }^{89}$.

Si le bilan des entrées et sorties de biens et de revenus publics pour l'époque carolingienne est voisin dans sa structure de celui de la période lombarde, l'infléchissement en faveur des prélèvements indirects vaut d'être noté. Omniprésents, bien mieux documentées que pour la période lombarde, ils sont aussi, semble-t-il, plus lourds. De même, le fait que l'on cherche ici et là à définir le contenu du ius fisci des temps carolingiens avec une précision inégalée est en soi nouveau. Reste que la mise en place et le développement de l'immunité ont dans bien des cas annulé les effets de ce qui, au moins durant la première génération qui a suivi la conquête, fut un durcissement.

Sur le plan foncier, l'affectation de terres rémunère comme auparavant le service et la fidélité, avec la même différence entre un circuit lent et un circuit court, ce dernier alimenté par les confiscations ${ }^{90}$. Au chapitre des entrées, il faut ajouter ceci de nouveau, que les biens du roi ont pu s'accroître par le biais du don immédiat ou différé. Le connétable Adalbert destinait ainsi à Louis II tout ce qu'il possédait en «Italie » (la plaine du Pô), en Toscane, dans le duché de Spolète et en Romania (l'ancien exarchat de Ravenne). Cependant, l'empereur les lui rendit en usufruit viager, en prévision d'un transfert, à terme, au profit de Saint-Pierre ${ }^{91}$. En Tuscie lombarde, d'autres biens furent per cartulam delegati à Louis II ; celui-ci les affecta à sa

pratiques sociales de l'anathème et de l'excommunication (IVe-XII siècle), Rome, 2015 (Haut Moyen Âge, 23), p. $215-238:$ p. 224.

${ }^{87}$ Placiti, I, n 56 ; MGH DD L II 4.

${ }^{88}$ Supra, texte correspondant à la note 56.

${ }^{89}$ Sur toute l'affaire, A. A. Settia, «L'età carolingia e ottoniana », in G. Andenna (éd.), Storia di Cremona. Dall'alto Medioevo all'età communale, Crémone, 2004, p. 38-105 : p. 41-52.

${ }^{90}$ MGH DD Kar. I 112, 187, 208, 214 ; DD L I 101 : biens confisqués à des otages, à des fugitifs chez les Avars ou à des partisans du rebelle Rotgaud; Placiti, I, no 32, et MGH DD L I 98, 140, DD Lo I 24 et DD L II 63 : attribution à Farfa, au Mont-Cassin et à Casauria de biens confisqués à des infidèles passés du côté de Bénévent ; ChLA XXXVIII, $\mathrm{n}^{\mathrm{o}} 1112$ (Lucques, 787) : une vente de biens est assortie d'une clause de réserve, au cas où le vendeur viendrait à perdre les biens par confiscation après avoir été cité en justice par la curtis regia (quod absit, si G. rem suam perdiderit et ad publicum devolutam fuerit...); Placiti, I, $\mathrm{n}^{\circ} 76$, a. 873 : confiscation au profit de la pars palatii des biens de Gundi, veuve remariée en dépit du fait qu'elle avait pris le voile ; etc. Voir Bougard, La justice, p. 245-246.

${ }^{91}$ Placiti, I, n 65, p. 236 ; MGH DD L II 99. 
fondation de la Sainte-Trinité de Casauria ${ }^{92}$. Signalons encore cette clause, dans une donation post mortem de l'évêque de Padoue à l'église cathédrale S. Giustina, en 874 : s'il venait à se raviser, ou si son successeur voulait affecter les biens autrement, ceux-ci passeraient aux mains du roi d'Italie en charge ${ }^{93}$.

Comme à l'époque lombarde, la disponibilité en métal précieux, orfévré ou non, a pu être alimentée par le butin, par exemple celui pris sur les Avars, dont on peut supposer, bien qu'il eût été envoyé à Aix-la-Chapelle, qu'une part échut à Pépin, qui conduisit la campagne militaire de 796. Il est probable, aussi, que l'expédition de Louis II en Italie méridionale a apporté son lot de prises. De même, la reconnaissance de la suprématie franque par le prince de Bénévent s'est exprimée, pendant quelques années au moins, par le versement d'un tribut, dont on peut supposer que le roi d'Italie a profité au moins en partie ${ }^{94}$. Ce sont autant d'occasions d'enrichissement du trésor royal, et de possibilités offertes de redistribuer cette richesse mobilière par le canal du don. À l'inverse, il a fallu parfois payer des sommes importantes, comme ce fut très probablement le cas pour la libération de Louis II et Engelberge en 871. De telles recettes et dépenses occasionnelles ne remettent pas plus en cause l'équilibre général que ne le font les cadeaux d'ambassade envoyés ou reçus. En revanche, avec les réformes monétaires de Charlemagne, le trésor royal italien n'a plus disposé comme auparavant de ce qui pouvait être perçu sur la frappe de la monnaie d'or. Cette situation est certes partagée avec celle du reste de l'Empire. Mais la moindre disponibilité de minerai en Italie introduisait un facteur de différenciation avec le nord des Alpes, en défaveur du regnum ${ }^{95}$.

La recherche d'équilibre, rendue d'autant plus nécessaire du fait de la diffusion des concessions en bénéfice, s'est traduite comme au nord des Alpes par la mise à disposition d'une partie des biens d'Église pour alimenter la distribution, ce qui était une manière de garder la main sur ce qui était par ailleurs donné ou concédé temporairement aux représentants de Dieu, au risque pour ces derniers de perdre assez vite le contrôle de ces éléments de leur patrimoine : ainsi à Bobbio, au Monte Amiata, à Pistoia, à Reggio, à S. Marino et S. Giovanni Domnarum de Pavie, à S. Salvatore de Brescia ${ }^{96}$. Les autorités ecclésiastiques du royaume s'en sont plaintes

\footnotetext{
${ }^{92}$ MGH DD L II 64 et p. 56-58 pour les actes documentant les acquisitions de Louis II passées ensuite à Casauria ; L. Feller, Les Abruzzes médiévales; Territoire, économie et société en Italie centrale du ix au xiie siècle, Rome, 1998 (Bibliothèque des Écoles françaises d'Athènes et de Rome, 300), p. 170-172.

${ }^{93}$ A. Gloria, Codice diplomatico padovano dal secolo sesto a tutto l'undecimo, Venise, $1877, \mathrm{n}^{\mathrm{o}} 15$ : in potestatem regis qui pro tempore Italia regere videtur.

${ }^{94}$ Annales regni Francorum, éd. F. Kurze, MGH SS rer. Germ. 6, a. 812 et 814, p. 137 et 141 : tributs de vingtcinq mille sous d'or, puis de sept mille sous annuels, imposés par Charlemagne puis par Louis le Pieux à Grimoald IV.

95 A. Rovelli, «Mines et monnaies au haut Moyen Âge. Les sources de l'Italie carolingienne », Bulletin de la Société française de numismatique, 59, 2004, p. 58-64.

${ }^{96}$ Bobbio : M. Nobili, « Vassalli su terra monastica fra re e 'principi' : il caso di Bobbio (seconda metà del sec. Xinizi del sec. XI » [1980], in Id., Gli Obertenghi e altri saggi, Pérouse, 2006, p. [299-309] 113-124 : p. [300-301] 114-115. En 862, une forêt rattachée à un xenodochium du monastère mais affectée à la pars du comte Boniface est considérée comme «perdue »: Inventari altomedievali di terre, coloni e redditi, p. 144. — Monte Amiata : MGH DD L II 11 : le monastère a été confié ad regendum au marquis Adalbert. — Pistoia : Placiti, I, n ${ }^{\circ} 25$ (supra, texte correspondant à la note 59). — Reggio : MGH DD Lo 140 : deux curtes prélevées à l'église de Reggio pour les affecter en bénéfice à un fidèle de Lothaire. — S. Marino de Pavie : MGH DD Kar. III 42. — S. Giovanni Domnarum : Éginhard, Translatio et Miracula ss. Marcellini et Petri, 6, MGH SS, 15/1, p. 242. - S. Salvatore de Brescia : C. La Rocca, « Les cadeaux nuptiaux de la famille royale en Italie », in F. Bougard, L. Feller et R. Le Jan (dir.), Dots et douaires dans le haut Moyen Âge, Rome, 2002 (Collection de l'École française de Rome, 295), p. 499-526: p. 506-507. - À Lucques, en 845, l'ancien comte Agano, qui venait d'être remplacé au profit d'Adalbert, reçut l'église $S$. Michele in Foro de la part de l'évêque, pendant cinq ans, moyennant le paiement d'un cens, à condition de rendre le bien si entretemps il en obtenait un autre en bénéfice de la part du roi ; l'acte ne dit pas dans quel type de patrimoine, public ou ecclésiastique, serait éventuellement taillé ledit bénéfice (Memorie e documenti per servire all 'istoria del Ducato di Lucca, V, 2, éd. D. Barsocchini, Lucques, 1837, nº 628 ; sur le rôle d'Agano, dont la charge fut une parenthèse politique dans la longue gestion du comté par la même famille d'origine
} 
auprès de Louis II au début de son règne, au motif que ces distributions les empêchaient de pourvoir aux utilitates des églises ${ }^{97}$. De même, ce qui relève de la chose militaire repose sur les détenteurs de la terre indépendamment de son statut d'alleu ou de bénéfice. Comme au VIII ${ }^{\mathrm{e}}$ siècle, aussi, on a tenté de lutter contre la déperdition insensible de revenus liée à la libre circulation de terres fiscales et au risque qu'elle entrainait, à la faveur des changements de possesseurs, de voir peu à peu se perdre le lien avec «l'État». Un capitulaire de Louis le Pieux daté de 818-819 et qui fut diffusé en Italie, prescrit ainsi que l'église ou l'individu qui viendrait à entrer en possession (par le jeu du marché ou des successions) d'une terre versant « tribut » à la partie publique doit continuer à s'en acquitter, sauf exonération explicite ${ }^{98}$.

La prescription de soixante ans pour les biens publics est toujours en vigueur. À cette protection s'ajoute la diffusion de la procédure par enquête en cas de litige les concernant. Cet héritage du Bas-Empire transmis par la juridiction lombarde fut particulièrement apprécié par les responsables carolingiens, qui en ont fait bénéficier la plupart des grands monastères et des églises épiscopales, dont les biens se trouvaient ainsi alignés sur ceux des res domnicatae ${ }^{99}$. Enfin, la vigilance des représentants de la pars regia, ou pars palatii, vaut d'être relevée. Certes, elle change de point de vue au fil du temps : après les décennies où nous les avons vus opérer de manière agressive, au mépris souvent des avantages acquis, les voici plutôt cantonnés à un rôle d'observateurs défiants face à la mise en œuvre des largesses des souverains, remettant en cause le bien-fondé des diplômes quand ils estiment lésés les intérêts du fisc. Les exemples dont nous disposons montrent surtout l'échec de telles résistances ; mais rien n'assure, compte tenu des canaux par lesquels a été transmise la documentation, qu'ils sont représentatifs de l'ensemble ${ }^{100}$.

III

Pour qui voudrait dresser le tableau de la nature et de la localisation des biens publics au $\mathrm{IX}^{\mathrm{e}}$ siècle, et sachant qu'il s'agit, pour l'essentiel, d'un héritage, il faudrait prendre en compte :

a) les éléments du paysage dits "publics ». Certains sont susceptibles de fournir un revenu sous forme de taxe, comme les cours d'eau ou les ponts. Ces derniers justifient aussi des prestations de service pour leur entretien de la part des hommes libres, en tant qu'il s'agit d'une obligation militaire $^{101}$. D'autres sont laissés à l'usage de la collectivité, comme la voirie (via, strata publica, parfois concédée sur une partie de son tracé, comme ce fut le cas à Plaisance pour permettre la construction de l'abbaye $\mathrm{S}$. Sisto ${ }^{102}$, et comme ce le fut régulièrement par la suite au fil des entreprises de fortification), certains gualdi ou certains espaces de pêche, par exemple le mare publicum dont les habitants de l'Istrie disent avoir eu la libre disposition jusqu'à la mise en place du pouvoir carolingien ${ }^{103}$ : c'est-à-dire autant de lieux où se cristallise

bavaroise, A. Castagnetti, «I vassalli imperiali a Lucca in età carolingia », in S. Pagano et P. Piatti (éd.), Il patrimonio documentario della chiesa di Lucca. Prospettive di ricerca, Florence, 2010, p. 211-284 : p. 234-236).

${ }^{97}$ MGH Capit. II, $\mathrm{n}^{\mathrm{o}} 210$, c. 10.

${ }^{98}$ MGH Capit. I, no 140, p. 287. Le texte a alimenté un débat sur le statut de la terre «tributaire ».

${ }^{99}$ F. Bougard, La justice dans le royaume d'Italie de la fin du VIII siècle au début du XI siècle, Rome, 1995, p. 194-203 ; S. Esders, « Die römischen Wurzeln der fiskalischen Inquisitio der Karolingerzeit », in C. Gauvard (éd.), L'enquête au Moyen Âge, Rome, 2008 (Collection de l'École française de Rome, 399), p. 13-28 ; J. Davis, Charlemagne's Practice of Empire, Cambridge, 2015, p. 259-278.

100 Placiti, I, n ${ }^{\text {os }} 91,103$.

${ }^{101}$ Inventari altomedievali di terre, coloni e redditi, p. 156-157. Tabacco, I liberi del re, p. 100-104.

${ }^{102}$ MGH DD II 67 : concedimus... ubicumque voluerit, infra ipsam urbem Placentinam vel circumcirca, publicas stratas... immutare.

${ }^{103}$ Placiti, I, no 17 : mare publicum, ubi omnis populus communiter piscabant. 
l'idée d'un bien commun, susceptible de s'exprimer dans bien des conflits à la fin du VIII et au $\mathrm{IX}^{\mathrm{e}}$ siècle. Un cas bien étudié mit aux prises la communauté de Flexo avec le monastère de Nonantola ${ }^{104}$. Au $\mathrm{X}^{\mathrm{e}}$ siècle encore, à Crémone, la terre qui dépend du gastaldiaticum, c'est-àdire du gastald de la curtis royale de Sospiro, fait partie des comunalia ${ }^{105}$. À cela se rattachent les terres dites des arimanni;

b) ce qui, en ville, est dit «du roi » : les murs d'enceinte (à Plaisance, à Milan), les espaces ouverts (par exemple à Arezzo, le forum où est rendue la justice ${ }^{106}$ );

c) tout ce qui, dans les désignations de confins dont regorgent les actes privés, est dit terra, casa, campus, silva vinea, pascua ou prata regis, avec une quantité de mentions telle qu'elle indique une présence capillaire de ces biens sur le territoire. Il faut y ajouter la terre dite « de la reine », pour laquelle les mentions sont peu abondantes mais suffisantes pour rappeler qu'elle existe, au reste dès l'époque lombarde ${ }^{107}$, et que, dès Charlemagne, la reine - Hildegarde - a disposé de plusieurs curtes en Italie, dont Pépin fit faire l'inventaire ${ }^{108}$. Une enquête plus fine sur la répartition des mentions de la terre royale permettrait d'en dire davantage. Dans le comté de Plaisance, par exemple, étudié par Giorgia Musina, la plupart des curtes fiscales se trouvent dans la basse plaine du Pô, et aux premières loges pour avoir probablement promu la bonification de l'inculte. Mais il s'agit tout au plus d'une situation ancienne, celle d'un fisc omniprésent dans l'incultum ${ }^{109}$.

Ces indications éparses se rapportent aux curtes et villae distribuées dans les comtés, confiées à tel ou tel et dont le revenu est ordinairement affecté aux regis expensa - c'est-à-dire dirigé vers la capitale, en monnaie ou en nature -, selon la formulation d'un capitulaire traditionnellement attribué à Lothaire et à l'année 832 , mais qui est plus probablement dû à Louis II, avant son accession à l'Empire $(844-850)^{110}$. Les inventaires relatifs à la curtis de Limonta au temps où celle-ci ne faisait pas partie du patrimoine de $\mathrm{S}$. Ambrogio de Milan mettent précisément en lumière, pour un domaine spécialisé dans l'oléiculture, non seulement la question de la production, mais aussi celle du transport à Pavie ${ }^{111}$.

d) les palais ruraux sont un élément nouveau par rapport à la période lombarde. Celleci avait légué les palais de Pavie, Ravenne, Milan, Vérone, Monza et Corteolona. En ville, les souverains carolingiens ont ajouté Mantoue et peut-être Turin. Mais leur apport réside surtout dans la promotion de quelques domaines ruraux (sept, outre celui de Corteolona: Auriola,

\footnotetext{
${ }^{104}$ Placiti, I, n ${ }^{\text {o }} 36$; T. Lazzari, « Comunità rurali nell'alto medioevo: pratiche di descrizione e spie lessicali nella documentazione scritta », in P. Galetti (éd.), Paesaggi, comunità, villaggi medievali, Spolète 2012, p. $405-421$ : p. 414 et suiv., avec l'abondante bibliographie sur l'affaire.

105 E. Falconi, Le carte cremonesi dei secoli VIII-XII, I, Crémone, 1979, nº 64 ; Settia, « L'età carolingia e ottoniana », p. 89.

${ }^{106}$ Plaisance : MGH DD L II 67 ; DD Kar. III 29 (murum regium). — Milan : L. Schiaparelli, I diplomi di Guido..., Rome, 1906 (Fonti per la storia d'Italia, 36), no 3. - Arezzo : G. Tessier, Recueil des actes de Charles le Chauve, roi de France (840-877), Paris, 1943-1955, n 404 (forum quod muro adiacet... ubi actenus mallationum ventilatae sunt lites).

${ }^{107}$ Pour la période lombarde, voir A. Ghignoli, Carte dell'Archivio arcivescovile di Pisa. Fondo arcivescovile, 1 (720-1100), Pise, 2006, nº 10/53 (= ChLA XXVI, no 808) ; Brühl, Fodrum, p. 366, 376. Pour le IX ${ }^{\mathrm{e}}$ siècle, Placiti, I, no 44, a. 840 (à Lucques) ; Ghignoli, Carte, no 29 (=ChLA ${ }^{2}$ LVIII, Dietikon-Zurich, 2011, n 12), a. 883. Voir aussi les toponymes, par ex. dans le territoire de Vérone, en 973 : Padulae quae dicitur Reginae... locus ubi dicitur Reginae (Codex diplomaticus Langobardiae, éd. G. Porro Lambertenghi, Turin, 1873, $\mathrm{n}^{\circ}$ 745).

${ }^{108}$ MGH Capit. I 95 (a. 781), c. 14.

${ }^{109}$ Musina, Le campagne di Piacenza, p. 150.

${ }^{110}$ MGH Capit. II 202, c. 6 : inquirant missi nostri (per singulos comitatus) villas et cortes, unde regis expensa ministrari solita sit, et a quibus personis modo detineantur. Brühl, Fodrum, p. 434-435. Pour la datation du texte, M. Geiselhart, Die Kapitulariengesetzgebung Lothars I. in Italien, Frankfurt am Main, 2002 (Freiburger Beiträge zur mittelalterlichen Geschichte, 15), p. 201-205.

${ }^{111}$ Inventari altomedievali di terre, redditi e coloni, p. 19-25, avec bibliographie ; nouvel examen de la datation des documents par A. Castagnetti, Una famiglia di immigrati nell'alta Lombardia al servizio del regno (846-898), Vérone, 2004, p. 112-119.
} 
Gardina, Marengo, Orba, Sospiro, Senna Lodigiana, Marmoriolo) au rang de palatium ${ }^{112}$. Adaptée aux exigences des déplacements de la royauté dans la plaine du Pô, leur localisation répond à des critères qui combinent la proximité avec les grandes voies de communication, les centres de décision et les réserves de chasse.

S'il fallait établir une hiérarchie entre ces trois critères, il est probable que la chasse l'emporterait : c'est elle qui peut rendre compte, notamment, de la présence de palatia très proches les uns des autres, comme jumelés (Auriola et Gardina, Orba et Marengo), indépendamment de tout souci d'une couverture raisonnée du territoire ${ }^{113}$. Par ailleurs, si la désignation palatium n'est pas seulement un fait de vocabulaire, il faut penser que les sites correspondants se distinguent par quelque élément matériel - un bâtiment de pierre, une salle de réception, comme c'est le cas à Marengo, voire un édifice plus imposant flanqué de tours, selon l'hypothèse faite à propos de Gardina $a^{114}$ - des autres curtes où le roi a séjourné. Ainsi forment-ils un groupe restreint au sein de l'ensemble plus vaste des domaines qui, d'après la distinction du capitulaire de 844-850 déjà cité, sont mobilisés non pour la « dépense du roi », mais pour les déplacements de l'empereur et ceux de ses missi $i^{115}$.

Il est difficile d'aller au-delà du constat de la répartition des revenus des domaines ruraux en fonction des postes de dépense telle que l'indique le capitulaire de 844-850, dans ce que l'on devine malgré tout constituer un réseau. Sa hiérarchie a peut-être hérité de la distinction déjà signalée entre ce qui relève du «public » et ce qui relève du palais, et dont on trouve encore l'écho dans un diplôme de Louis II, qui fait état de palatia et principales coortes dans le comté de Plaisance, en $874^{116}$. Quoi qu'il en soit, dans l'ensemble particulier que forment les palais, il n'est guère possible, en l'absence d'enquête archéologique systématique, de repérer une éventuelle logique économique liée à la production de telle ou telle curtis en particulier. L'exploitation des ressources aurifères des sables fluviaux a pu jouer un rôle ici ou là, d'autant plus important que le Palais avait le monopole sur les acquisitions de métal précieux, tout au moins au $\mathrm{X}^{\mathrm{e}}$ siècle ${ }^{117}$. À Orba, la présence de vaisselle en «pietra ollare » témoigne d'une bonne insertion dans les circuits commerciaux liés à l'arc alpin, sans plus de différence, toutefois, par rapport à de nombreux autres sites de plaine qui recevaient de la même manière la production artisanale des hautes vallées ${ }^{118}$. Quant à la maîtrise de grandes étendues forestières et plus généralement foncières, elle est essentielle pour la définition du palais rural, mais non suffisante, si l'on songe par exemple que la curtis de Benevagienna, dans le Piémont, comptait cent mille jugères sans pour autant être comptée parmi les palais ${ }^{119}$;

e) l'autre nouveauté est l'existence d'un fisc comtal. Si l'expression fiscus comitalis est bien attestée à la fin du $\mathrm{IX}^{\mathrm{e}}$ siècle $^{120}$, savoir ce qu'elle recouvre n'est pas toujours aisé, car la seule mention d'une terra comitis ou d'une curtis ducale au $\mathrm{IX}^{\mathrm{e}}$ siècle ne permet pas de faire le départ entre ce qui relèverait des biens privés et de ceux afférents à la fonction. Certaines

\footnotetext{
${ }^{112}$ F. Bougard, « Palais royaux et impériaux de l'Italie carolingienne et ottonienne », in A. Renoux (dir.), Palais royaux et princiers au Moyen Âge, Le Mans, 1996, p. 181-196; la carte a été à nouveau publiée dans C. La Rocca (éd.), Italy in the Early Middle Ages 476-1000, Oxford, 2002, p. 261. La localisation d'Auriola et de Gardina, en Piémont, a été précisée depuis : A. A. Settia, « Nelle foreste del re : le corti Auriola, Gardina e Sulcia dal IX al XII secolo », in Vercelli nel secolo XII. Atti del quarto congresso storico vercellese, Vercelli, 2005, p. 353-410.

${ }^{113}$ Settia, «Nelle foreste del re », p. 398, relève la présence d'un venator royal à Verceil, proche d'Auriola et Gardina, au X ${ }^{\mathrm{e}}$ siècle : L. Schiaparelli, I diplomi di Ugo e di Lotario..., Rome, 1924 (Fonti per la storia d'Italia, 38), $\mathrm{n}^{\mathrm{o}} 73$.

${ }^{114}$ Placiti., III, no 358 ; Settia, « Nelle foreste del re », p. 399-402.

115 MGH Capit. II 202, c. 6 : necnon et (villae et cortes) quae in transitu domni imperatoris servire debent vel missis transeuntibus necessaria ministrare.

${ }^{116}$ MGH DD L II 67 ; supra, texte correspondant aux notes 20 et 48.

${ }^{117}$ Brühl \& Violante, Die “Honorantiae civitatis Papie”, p. $21 \S 9$.

118 M. Cortelazzo, in Archeologia medievale, 20, 1993, p. 339-345.

${ }^{119}$ L. Schiaparelli, I diplomi italiano di Ludovico III..., Rome, 1910 (Fonti per la storia d'Italia, 37 ), n 13.

${ }^{120}$ MGH Capit. II 225, c. 8 ; infra, note 146.
} 
formulations lèvent toutefois le doute. En 826, Louis le Pieux et Lothaire font ainsi un échange avec le comte Boson, lui donnant des biens qui «appartiennent» (pertinentes) au comté de Verceil $^{121}$. En 876, l'archevêque de Milan reçoit de Charles le Chauve des casellae dépendant du comté de Pavie, mais sises dans le territoire de la ville de Milan ${ }^{122}$. En 878, voici encore une donation de biens iuris publici de comitatu Placentino à S. Sisto de Plaisance ${ }^{123}$. Et en 894 l'évêque de Parme Wibod se voit confirmer l'attribution d'une curtis dans le territoire de Parme (in finibus) avec cette précision : sed aliud quod ibi pertinet ad comitatum ${ }^{124}$. Le verbe pertinere doit être considéré dans sa pleine acception juridique, à la différence de la simple localisation topographique in comitatu (posito, ou adiacens). Un diplôme de Lambert de Spolète l'exprime encore à propos de biens sis in comitatu et pago Placentino, mais dont il est précisé qu'ils sont de eodem pertinentes comitatu ${ }^{125}$.

Pour autant, ces distinctions, souvent utiles comme signes du caractère dispersé de la propriété, ne préjugent pas du fait que ce qui relève du comté reste soumis au ius regni (nostri), ou ius nostrum publicus, comme le disent de nombreux diplômes ${ }^{126}$. C'est pourquoi il serait hasardeux de penser que ce qui ressort du fisc comtal forme une entité séparée, car cet élément s'intègre sans difficulté dans l'une ou l'autre des catégories définies par le capitulaire de 844850.

IV

À la fin du $\mathrm{IX}^{\mathrm{e}}$ et pendant la première moitié du $\mathrm{X}^{\mathrm{e}}$ siècle, ce système est maintenu dans ses grands traits mais connaît aussi des infléchissements notables, liés au contexte politique.

Dès les années 860 , la constitution du douaire d'Engelberge ${ }^{127}$ et l'abondante série de diplômes qui ont suivi en sa faveur portent en germe les prémices de certains dérèglements. Le fait que la reine dispose de biens propres relève en soi d'une pratique courante, nous l'avons vu, depuis l'époque lombarde. Hildegarde eut aussi ses curtes italiennes. De même le monastère S. Salvatore de Brescia fut-il attribué d'office à la souveraine en exercice : même Judith, qui n'a jamais mis les pieds en Italie, en a eu le beneficium ${ }^{128}$. Ce ne sont même pas le nombre, ni l'étendue des biens concernés qui sont en cause, puisque seules deux curtes sont envisagées dans le douaire au sens strict, établi en 851/860. Louis II avait par ailleurs pris soin de recueillir l'accord des grands avant de les céder: consensus nécessaire, peut-on penser, puisqu'il s'agissait précisément de biens publics pour lesquels le roi ne peut agir de manière complètement arbitraire et qui, par définition, ne pouvaient faire l'objet d'une cession définitive $^{129}$. Le malaise vient du fait que, une donation après l'autre, ce dotalicium s'est transformé en tout autre chose, hors norme.

Surtout, dans les dernières années du règne de Louis II et à la faveur de la crise politique qui a suivi son décès, tout fut fait pour soustraire ces biens à la sphère publique ordinaire en les

\footnotetext{
${ }^{121}$ MGH DD L I 256.

122 Tessier, Recueil des actes de Charles le Chauve, $\mathrm{n}^{\circ} 402$ : quasdam casellas ad comitatum Papiensem pertinentes, quae reiacent in finibus praefatae Mediolanensis urbis.

${ }^{123}$ MGH DD Kn 16.

${ }^{124}$ MGH DD Arn 125.

${ }^{125}$ L. Schiaparelli, I diplomi di... Lamberto, Rome, 1906 (Fonti per la storia d'Italia, 36), no 1.

126 Un seul exemple parmi beaucoup d'autres, L. Schiaparelli, I diplomi di Berengario I, Rome, 1903 (Fonti per la storia d'Italia, 35), $\mathrm{n}^{\circ} 16$ : donation d'un manse iuris regni nostri, pertinentem de comitatu Veronense.

${ }^{127}$ R. Cimino, «Angelberga : il monastero di San Sisto di Piacenza e il corso del fiume Po », Reti Medievali Rivista, 13, 2, 2012 (http://rivista.retimedievali.it), p. 141-162.

${ }^{128}$ MGH DD L I 246 ; C. La Rocca, « Les cadeaux nuptiaux de la famille royale en Italie », in F. Bougard, L. Feller et R. Le Jan (éd.), Dots et douaires dans le haut Moyen Âge, Rome, 2002 (Collection de l'École française de Rome, 295), p. 499-526: p. 506.

${ }^{129}$ MGH DD L II 30 : una per consensum et voluntatem nostrorum optimatum.
} 
transférant à la fondation d'Engelberge, S. Sisto de Plaisance. En 874, l'empereur autorisa sa consors imperii à disposer librement de la totalité de ses biens, y compris de ceux provenant de sa « libéralité » ${ }^{130}$. Quelque temps plus tard, un faux fut composé à S. Sisto pour dire la même chose, mais en rétrodatant la concession à l'année 870 et en prenant soin d'intégrer la mention de l'accord unanime des primores, avec des mots autrement plus forts que dans l'acte de $860 / 851^{131}$. La nécessité d'établir un diplôme pour cela, puis d'en renforcer la teneur par le biais d'une falsification, est révélatrice du fait que cela n'allait pas de soi et qu'une telle faveur, inédite, ne pouvait que susciter des résistances. Celles-ci n'ont pas manqué. En 887, un diplôme de Charles le Gros évoque les «contradictions » dont est victime Engelberge, à commencer pour ce qui est des biens reçus de son époux ${ }^{132}$. L'identité de tels contradicteurs n'est pas difficile à établir: en 877, Charles le Chauve avait fait mis main basse sur le trésor de l'impératrice, déposé à S. Salvatore de Brescia ; l'année 879, elle, fut marquée par l'offensive de deux comtes et de l'évêque de Novare contre des biens fonciers. Autant de comportements que le pape, protecteur de la veuve de Louis II, fustige comme de la déprédation ${ }^{133}$. Mais ne s'agit-il pas plutôt de la réaction normale de qui, après la mort de l'empereur, considérait que les biens confiés à son épouse, prélevés sur les fiscs au sein de différents comtés, pouvaient faire retour dans les caisses publiques ? Il n'y a pas de hasard non plus si Engelberge a tenu à se faire confirmer ses biens par tous les souverains successifs : non seulement Charles le Chauve (en dépit des appétits de ce dernier sur le trésor), Carloman, Charles le Gros, Bérenger $\mathrm{I}^{\mathrm{er}}$ et Gui de Spolète, mais aussi Louis le Germanique en 876 puis Arnulf en 889, alors que ces derniers n'étaient en rien maîtres de l'Italie mais s'imposaient comme des acteurs puissants, maîtres en puissance du fisc italien, à ne pas négliger ${ }^{134}$.

Tout se passe comme si, au prix d'une négociation permanente, on avait voulu changer les règles du jeu (en gros, celles de l'usufruit viager), pour éviter que ces biens d'origine publique ne soient réinjectés dans le circuit ordinaire de la distribution rémunératrice, qui comportait les mêmes risques d'érosion qu'à l'époque lombarde, amplifiés qui plus est par la tendance à la possession héréditaire des bénéfices. Le fait que $S$. Sisto ait par la suite été confié à des représentantes de la famille royale (spécialement Berthe, la fille de Bérenger $\mathrm{I}^{\mathrm{er}}$, qui disposait aussi de S. Salvatore de Brescia), laissait ces terres sous le contrôle de la royauté ${ }^{135}$. Celle-ci put en distraire tel ou tel élément pour constituer d'autres douaires, comme celui d'Ageltrude, dont la position patrimoniale fut renforcée en 891, en lien direct avec l'accession

\footnotetext{
${ }^{130}$ MGH DD L II 66.

${ }^{131}$ MGH DD L II 51 : cum tractatu et consilio atque unanimitate imperii nostri primorum. L'acte, que l'éditeur considère comme interpolé, est intégralement rejeté, à juste titre selon moi, par (J. F. Böhmer)-H. Zielinski, Regesta imperii, I, 3, 1, Cologne-Vienne, 1991, nº 307.

132 DD Kar. III 166.

${ }^{133}$ MGH Epp. VII, $n^{\text {os }} 181,238,244$. Voir aussi no 173 : le comte Liutfrid et son épouse ont retiré de force du monastère S. Sisto la moniale Garelinda : probablement s'agissait-il de leur fille, qu'il n'y avait plus d'intérêt à laisser auprès d'Engelberge, alors en mauvais termes avec Charles le Gros.

${ }^{134}$ MGH DD Kar. III 22 (qui fait état d'un diplôme de Carloman, perdu), 56, 166 (qui fait état d'un diplôme de Charles le Chauve, perdu) ; Schiaparelli, I diplomi di Berengario I, $\mathrm{n}^{\circ} 4$; Id., I diplomi di Guido, $\mathrm{n}^{\circ} 4$; DD LD 171; DD Arn 49. Les confirmations en faveur de S. Sisto postérieures à la mort d'Engelberge montrent que l'ensemble qu'elle avait constitué fut peu affecté par la remise en circulation de biens au profit des reines ou d'autres bénéficiaires : L. Schiaparelli, I diplomi italiani... di Rodolfo II, Rome, 1910 (Fonti per la storia d'Italia, 37), $\mathrm{n}^{\circ} 8$; Id., I diplomi di Ugo e di Lotario, di Berengario e di Adalberto, nº 2, p. 6 ; n 1, p. 291.

${ }^{135}$ C. Sereno, «Bertilla e Berta : il ruolo di Santa Giulia di Brescia e di San Sisto di Piacenza nel regno di Berengario I », Reti Medievali Rivista, 13, 2, 2012 (http://rivista.retimedievali.it), p. 187-202 ; T. Lazzari, «Bertha, amatissima : Berengar I's daughter and the rule on the fical estates in the Po valley, $10^{\text {th }}$ century, first half », communication à l'International Medieval Congress 2017 (Leeds), à paraître.
} 
à l'Empire de Gui de Spolète ${ }^{136}$. En ce sens, l'expression «coffre-fort pour le royaume » est juste ${ }^{137}$.

Une telle mise en réserve pose aussi la question de l'usage des revenus qui lui sont liés : quelle est la contrepartie pour le fonctionnement même de la royauté ? Certes, les données du problème ne sont pas seulement économiques. Disposer d'un point d'appui à Plaisance, nœud de communications en plaine du Pô et commandant l'accès vers la Toscane, c'est-à-dire Rome, était un atout non négligeable. La possibilité de s'assurer une clientèle acquise aux intérêts du roi est primordiale. Mais cet aspect très politique, mêlant le souci de faire obstacle aux éventuelles appropriations par des individus qui pourraient se révéler hostiles à terme, tout en se ménageant une liberté de circulation, me paraît postérieure aux années de Louis II, c'est-àdire à la constitution de la dotation de $\mathrm{S}$. Sisto : elle correspond au veuvage d'Engelberge et à ses efforts pour peser sur le destin du royaume. Comme pour S. Salvatore de Brescia, qui a pu au reste servir de modèle, la dimension mémorielle et dynastique de la fondation ne doit jamais être oubliée. Quant à la lecture encore une fois «stratégique» de la localisation des biens fonciers de S. Sisto, elle me paraît surévaluée, en une région où il était difficile d'échapper à la proximité du Pô ou de l'un ou l'autre de ses nombreux affluents navigables.

La fondation personnelle de Louis II à Casauria, initialement dédiée à la sainte Trinité, n'a pas bénéficié des mêmes largesses fiscales que S. Sisto, en dépit de son emplacement, dont la portée politique, pour le coup, ne pouvait échapper à personne. Même si tout laisse croire que l'initiative même d'une fondation, qui suit immédiatement le retour dans l'espace du royaume à l'issue de la captivité de Bénévent (septembre 871$)^{138}$, relève de l'accomplissement d'un vœu, la position de cette nouvelle abbaye, à la limite méridionale du duché de Spolète, faisait d'elle une gardienne sur le versant adriatique comme le faisait Farfa du côté des terres romaines, et un pendant éventuel à S. Vincenzo al Volturno. La dotation initiale paraît avoir été constituée surtout à partir de ce que l'empereur avait pu obtenir par des achats, des dons ou des confiscations $^{139}$. En revanche, et peut-être précisément à cause de ce manque de ressources publiques importantes dans la région ou de leur méconnaissance au moment de la fondation, l'abbé semble avoir disposé de liquidités importantes, grâce auxquelles il put mener une politique foncière agressive, en bousculant la structure des patrimoines locaux ${ }^{140}$.

La compétition pour le trône, avec la nécessité qu'elle entraînait de devoir rallier des fidèles aux opinions parfois changeantes, s'est-t-elle par ailleurs traduite par une déperdition rapide, sinon massive de biens fiscaux ? La tendance est bien là, mais l'analyse doit être nuancée. Si le «bassin fiscal » dont disposent les concurrents n'est pas très grand, car il est rare que l'un ou l'autre ait la maîtrise concrète de la totalité du terrain, il ne semble pas que cela ait eu pour conséquence une pression plus forte, car les partisans des uns et des autres étaient répartis à peu près équitablement sur le terrain. Certes, les diplômes en faveur d'individus sont plus nombreux. Mais si on les rapporte à ceux qu'ont pu délivrer les rois lombards d'après l'exemple du gastald Alahis ${ }^{141}$, cette quantité devient infime. Par ailleurs, les biens concédés sont rarement de grande taille : on parle de terrola, corticella, casella etc. Il faut y voir une part de rhétorique, spécialement de la part de destinataires souvent à l'origine du texte même des diplômes et qui minorent volontiers la portée supposée de leur demande. Mais le fait est que,

\footnotetext{
${ }^{136}$ P. Guglielmotti, « Ageltrude : dal ducato di Spoleto al cuore del regno italico », Reti Medievali Rivista, 13, 2 , 2012 (http://rivista.retimedievali.it), p. 163-186.

137 T. Lazzari, « Dotari e beni fiscali », Reti Medievali Rivista, 13, 2, 2012 (http://rivista.retimedievali.it), p. 123138 : p. 137.

${ }^{138}$ Pour la datation, voir (J. F. Böhmer)-H. Zielinski, Regesta imperii, I, 3, 1, nº 333.

${ }^{139}$ Supra, texte correspondant aux notes 90 et 92.

${ }^{140}$ Feller, Les Abruzzes médiévales, p. 175-178 ; Id., A. Gramain et F. Weber, La fortune de Karol. Marché de la terre et liens personnels dans les Abruzzes au haut Moyen Âge, Rome, 2005 (Collection de l'École française de Rome, 347), p. 41-49.

${ }^{141}$ Supra, texte correspondant à la note 28.
} 
dans bien des cas, les dimensions paraissent, de fait, réduites. Il n'y a pas en tout cas de quoi parler de «dilapidation» du fisc en direction des personnes privées.

Les vrais bénéficiaires du transfert sont les églises épiscopales et, dans une moindre mesure, les monastères. Pour autant, sauf exception et sauf dans des pièces interpolées, les diplômes postérieurs à Louis II ne révèlent pas de concessions démesurées. On a depuis longtemps établi que des donations aussi exorbitantes que celle, prêtée à Carloman puis supposément confirmée par Charles le Gros, de la curtis regia de Parme en faveur de l'église locale, assortie du districtus sur la cité, étaient le fait des faussaires ${ }^{142}$. Les difficultés naissent plutôt de l'effet cumulé : a) des «transfusions » répétées du ius royal ou impérial vers d'autres ayants droit ; b) de l'immunité fiscale tantôt acquise depuis longtemps, tantôt complétée ou élargie, tantôt encore attribuée à de nouveaux acteurs, comme les chapitres canoniaux, et cela même si le privilège de l'immunité ne dispense généralement pas de fournir à l'entretien des $m i s s i^{143}$; c) de la concession de revenus de la fiscalité indirecte, qui relèvent tantôt du tonlieu sur les ports fluviaux, tantôt de la frappe de la monnaie (à Mantoue, Trévise), tantôt de ce que rapportent les foires et marchés nouvellement créés, spécialement en milieu rural, et qui représentent autant de manques à gagner ${ }^{144}$, tantôt encore de l'accompagnement obligé de concessions foncières - car on n'imagine plus guère, à partir des années 880 , que la donation d'une terre, quelle qu'elle soit, par le roi ne soit pas assortie des tonlieux qui lui sont attachés. Localement, des situations de crise amènent aussi à d'amples transferts de revenus qui valent compensation, comme en 916 au profit de l'église de Crémone, appauvrie par les incursions hongroises et qui bénéficia du produit des taxes non seulement en ville mais dans un rayon de cinq milles hors de celle-ci ${ }^{145}$.

Il existe bien une tension sur les biens publics à la fin $d u I^{\mathrm{e}}$ siècle. Le capitulaire promulgué en 898 à Ravenne par Lambert de Spolète en pose un diagnostic partiel - c'est-àdire ne prenant pas en compte ce qui est réservé pour les douaires -, en tentant de reprendre d'une main ce qui avait été donné de l'autre. Au moment de réaffirmer «l'ancienne coutume » de la fourniture du pastus imperatoris par les évêques et les comtes, au sein d'un programme politique qui se voulait un retour aux sources carolingiennes, on s'aperçut en effet que la dotation dont pouvaient disposer les comtes pour répondre à cette exigence avait pu fondre, ici et là, « récemment » (novo tempore). Le système décrit plus haut n'était plus à même de rendre le service pour lequel il avait été construit : d'où l'idée d'augmenter le stipendium impérial fourni par les églises, à proportion du transfert de biens publics relevant du fisc comtal en leur faveur, selon la même logique qui faisait tailler des bénéfices sur les patrimoines concédés aux hommes de Dieu ${ }^{146}$.

Il y a fort à parier que la mort de Lambert, survenue la même année, laissa à l'état de vœu pieux un tel mécanisme de compensation. De sorte que les ressources publiques se trouvaient affaiblies sur un double front, celui des disponibilités foncières et celui des rentrées

\footnotetext{
${ }^{142}$ MGH DD Kn 24 ; DD Kar. III 115 ; O. Guyotjeannin, « Les pouvoirs publics de l'évêque de Parme au miroir des diplômes royaux et impériaux (fin $\mathrm{IX}^{\mathrm{e}}$-début $\mathrm{XI}^{\mathrm{e}}$ siècle », in D. Barthélemy et J.-M. Martin (éd.), Liber largitorius. Études d'histoire médiévale offertes à Pierre Toubert par ses élèves, Genève, 2003, p. 14-31 : p. 23.

${ }^{143}$ La canonica de Reggio en est bénéficiaire, en 883 (MGH DD Kar. III 85) ; la même année, l'église de Bergame, immuniste de longue date, devient exempte des venationes publicae (DD Kar. III 89). Sur l'immunité et les missi, Brühl, Fodrum, p. 449-450.

${ }^{144}$ Schiaparelli, I diplomi di Berengario I, $\mathrm{n}^{\text {os }} 12,52,60,67,118$. A. A. Settia, «Per foros Italiae. Le aree extraurbane fra Alpi e appennini », in Mercati e mercanti nell'alto Medioevo : l'area euroasiatica e l'area mediterranea, Spolète, 1993 (Settimane di studio del CISAM, 40), p. 187-233.

145 Schiaparelli, I diplomi di Berengario I, $\mathrm{n}^{\mathrm{o}} 112$.

${ }^{146}$ MGH Capit. II 225, c. 8 : Ut pastus imperatoris ab episcopis et comitibus secundum antiquam consuetudinem solvatur. Quodsi novo tempore fiscus comitalis in ius ecclesiasticum concessus est, augeatur stipendium imperiale ab ecclesia, iuxta quod res publicae fuerint minoratae. Brühl, Fodrum, p. 445-447. Charles le Chauve avait précisément donné à l'archevêque de Milan des biens relevant du fisc comtal de Pavie : Tessier, Recueil, n 402 et supra, texte correspondant à la note 122 .
} 
indirectes, à un moment où il fallait non seulement financer la compétition politique mais, à partir de 899, payer régulièrement des tributs aux Hongrois, qui venaient s'ajouter à ce qu'exigeaient probablement les Sarrasins mais sur quoi nous sommes moins bien renseignés. En l'absence de données chiffrées, il serait assurément hasardeux de noircir le tableau. Mais certains ont pu être tentés, pour s'assurer des recettes, par l'alourdissement du prélèvement direct ou par le recours à une taxation extraordinaire. En 914, l'évêque de Parme Elbuncus, dans son testament, interdit que les biens légués à son église, parmi lesquels figurent de nombreux objets précieux, soient utilisés pour satisfaire aux dona regum: faut-il y voir l'expression d'une intolérance nouvelle face à des exigences répétées, en dehors du souhait légitime que le don à Dieu ne soit pas soustrait pour des usages non canoniques ? ${ }^{147}$ On se souvient que Bérenger I ${ }^{\text {er }}$, en 921, soumit l'accession de Lambert à la chaire archiépiscopale de Milan au versement d'une importante somme d'argent, qui fut ensuite répartie de manière hiérarchisée entre les serviteurs du palais de Pavie ${ }^{148}$. En 947, Bérenger II, lui, alla jusqu'à lever un impôt par tête pour réunir les dix muids de monnaies qu'il devait payer au chef hongrois, tout fondant les pièces pour les allier avec du cuivre, allégeant ainsi le coût de l'opération : occasion pour Liudprand de Crémone de filer une comparaison implicite avec Julien l'Apostat, qui avait taxé les chrétiens au prétexte d'une campagne militaire contre les Perses ${ }^{149}$.

Dans le deuxième quart du $\mathrm{X}^{\mathrm{e}}$ siècle, le règne de Hugues de Provence fut marqué par une reprise en main des biens et des revenus publics. Hugues est d'abord celui qui, dans un palais reconstruit par ses soins après l'incendie de Pavie de $924^{150}$, a remis sur pied l'organisation de la camera regis, qui fut confiée au magister Gisulfus. Les Instituta regalia et ministeria camerae regum Langobardorum, montrent le circuit d'une partie de la fiscalité indirecte et permettent surtout d'apprécier le poids qu'elle a pu représenter. Les taxes pèsent d'abord sur les marchandises, au passage des frontières alpines, avec une possibilité d'abonnement sous forme par exemple d'un versement tous les trois ans de métal et d'objets précieux, selon l'accord conclu avec les Anglo-Saxons. Elles touchent aussi le trafic des Vénitiens dans le royaume et ce qui arrive aux portes de la capitale depuis ce même royaume ou depuis les principautés méridionales. Plusieurs métiers sont aussi concernés : ceux de la pêche, du cuir, du savon, du transport fluvial ${ }^{151}$. Une bonne partie de cette description relève probablement d'un système ancien, hérité. En revanche, les données relatives à la monnaie ont toute chance d'être contemporaines de la réalité du $X^{\mathrm{e}}$ siècle. L'exigence de ne pas frapper de pièces au titre inférieur à 10/12 (soit 833 millièmes) correspond bien à ce qu'on sait de la qualité des deniers d'Otton $\mathrm{I}^{\mathrm{er}}(850 / 1000)$, bien supérieure à ceux du temps de Bérenger $\mathrm{I}^{\mathrm{er}}(746 / 1000)$. En l'absence d'analyses sur la production de Hugues, il reste difficile de savoir si l'amélioration constatée à partir d'Otton, que l'on explique volontiers par des raisons fiscales ${ }^{152}$, lui doit quelque chose. Notons toutefois que son attention pour la question monétaire, en elle-même

\footnotetext{
${ }^{147}$ E. Falconi, «Il testamento del vescovo Elbunco. Note sulla scrittura parmense nei secoli X e XI », Archivio storico per le province parmensi, $4^{\mathrm{e}}$ s., 9, 1957, p. 49-67.

${ }^{148}$ Liudprand de Crémone, Antapodosis II, 57.

${ }^{149}$ Liudprand de Crémone, Antapodosis V, 33 ; Cassiodore, Histoire tripartite VI, 39, 1-2 (voir ma traduction, Paris, 2015, p. 321 et 514).

${ }^{150}$ Placiti, I, $\mathrm{n}^{\circ} 136$ (a. 935) : in palacium noviter aedificatum ab domnum Uglonem gloriosissimum rex ; $\mathrm{n}^{\circ} 144$ (a. 945): in palacio domnorum regum ab eis noviter aedificatum. A. A. Settia, «Pavia carolingia e postcarolingia », in Storia di Pavia, II. L'alto medioevo, Pavie, 1987, p. 69-158: p. 105.

${ }^{151}$ Brühl \& Violante, Die "Honorantiae civitatis Papie"; pour le monopole sur les achats d'or issu du lavage des sables fluviaux, voir supra, texte correspondant à la note 117 .

${ }^{152}$ A. Saccocci, « La monetazione del Renum Italiae e l'evoluzione complessiva delsistema monetario europeo tra VIII e XII secolo », in C. Alfaro, C. Marcos et P. Otero (éd.), XIII Congreso internacional de numismática, Madrid 2003, Madrid, 2005, p. 1037-1049 : p. 1043.
} 
source de revenus, s'est traduite par la relance de l'atelier de Lucques, fût-ce de manière minime ${ }^{153}$.

Seul maître du terrain à partir de 926, Hugues put se permettre d'être plus parcimonieux que ses prédécesseurs en ce qui concerne la dévolution des biens fonds et des revenus issus du fisc $^{154}$. Sa concession la plus importante - mais elle est présentée comme une confirmation et ne devrait donc pas être considérée comme une aliénation - concerne l'église de Parme, en $926^{155}$. L'élimination systématique des opposants fut par ailleurs un moyen d'augmenter les ressources à la disposition du souverain. Il s'agit non seulement de terres, comme celles du marquis Adalbert « le Riche », récupérées en grande partie du fait de la mise à l'écart de ses deux fils, Gui et Lambert, mais aussi des trésors d'objets précieux, dont Liudprand de Crémone rapporte par exemple la quête à propos de Walpertus et Gezo, les deux juges de Pavie qui s'étaient conjurés contre Hugues, puis à propos de Willa, belle-sœur du roi, qui tenta en vain d'emporter dans sa fuite un certain baudrier orné de pierreries ${ }^{156}$. Pour servir sa politique, Hugues eut par ailleurs la possibilité de puiser dans les biens qui lui venaient de sa famille et dans ce qu'il avait pu recevoir par des donations ou autres transactions. En indiquant avec précision la différence dans l'origine de ses libéralités, les diplômes n'expriment pas une prise de conscience nouvelle de ce qui relève de l'individu par rapport au «transpersonnel ». Il s'agit simplement pour le nouveau roi, qui n'avait pas d'attache particulière dans la péninsule, d'exploiter au mieux ce qu'il y détenait, principalement par la voie de l'héritage de Berthe de Toscane, sa mère, et d'ajouter ainsi une dimension supplémentaire à la manifestation de sa générosité ${ }^{157}$.

La constitution des douaires «jumeaux» de Berthe et d'Adélaïde en 937, remarquablement étudiée par Giacomo Vignodelli, marque, elle, la volonté de s'assurer la maîtrise d'une large partie des biens fiscaux ${ }^{158}$. En rassemblant au profit de sa femme et de sa bru des ensembles patrimoniaux immenses dont il n'était pas question, cette fois, de préparer la dévolution à une fondation pieuse comme au temps d'Engelberge, Hugues se constituait une réserve foncière sécurisée tout en se donnant les moyens d'un meilleur contrôle du territoire, tant sur le plan des ressources économiques que sur celui des axes et nœuds de communication. Dans l'opération, qui implique les terres au voisinage de Pavie - principalement les curtes liées à la forêt d'Orba, mais aussi l'ancienne Olonna, dont l'importance pour la royauté n'est plus à démontrer -, la Toscane et la Lunigiana, le fait de puiser largement dans des biens situés en Toscane fait partie du train de mesures destinées à brider les tendances autonomistes de la marche. Pour autant, puisque Hugues disposait par ailleurs du réservoir formé par les biens

\footnotetext{
${ }^{153}$ Brühl \& Violante, Die "Honorantiae civitatis Papie”, § 7, p. 21. L. Travaini, « La moneta milanese tra X e XII secolo. Zecche e monete in Lombardia da Ottone I alla riforma monetaria di Federico Barbarossa », in Milano e il suo territorio in età comunale. Atti del $11^{\circ}$ Congresso internazionale di studi sull'alto medioevo, Spolète, 1989, p. 223-243: p. 227-228; A. Rovelli, «The Denaro of Pavia in the early Middle Ages (eighth to eleventh century) » [1995], in Ead., Coinage and Coin Use in Medieval Italy, Farnham, 2012 (Variorum Collected Studies Series, 1023), article VIII ; W. R. Day, Jr, M. Matzke et A. Saccocci, Medieval European Coinage, 12. Italy I (Northern Italy), Cambridge, 2016, p. 35.

${ }^{154}$ G. Fasoli, I re d'Italia (888-962), Florence, 1949, p. 216, fournit une estimation chiffrée : un diplôme sur cinq est concerné par des aliénations de terre royale sous le règne de Hugues, contre plus du tiers sous Bérenger $\mathrm{I}^{\mathrm{er}}$; de même, les aliénations de droits fiscaux ne représentent que $6 \%$ de ses diplômes, contre près du quart sous Bérenger.

${ }^{155}$ Schiaparelli, I diplomi di Ugo..., n 3 ; Guyotjeannin, « Les pouvoirs publics de l'évêque de Parme », p. 26-27.

${ }^{156}$ Liudprand de Crémone, Antapodosis III, 41 ; IV, 12.

${ }^{157}$ Schiaparelli, I diplomi di Ugo e di Lotario..., $\mathrm{n}^{\text {os }} 31-33,41,49,56,60,64,73$.

158 Schiaparelli, I diplomi di Ugo e di Lotario..., n ${ }^{\text {os }}$ 46-47. G. Vignodelli, «Berta e Adelaide : la politica di consolidamento del potere regio di Ugo di Arles» Reti Medievali Rivista, 13, 2, 2012 (http://rivista.retimedievali.it), p. 247-294 ; Id., «Reshaping a frame : the system of fiscal curtes in northern Italy and the politics of King Hugh of Arles (926-945) », communication à l'International Medieval Congress 2017 (Leeds), à paraître (je remercie l'auteur de m'avoir très tôt fait partager son texte). Les lignes qui suivent doivent tout à ces contributions.
} 
maternels, majoritairement situés en Toscane, il pouvait continuer à alimenter le circuit de la distribution.

La mobilisation des biens fiscaux par Hugues n'était pas seulement un retour à une politique peut-être plus raisonnée qu'auparavant. Elle est surtout guidée par la volonté de s'assurer un monopole, rendu évident par le fait que, partout où il l'a pu, Hugues a placé dans les douaires l'intégralité des domaines habituellement réservés au séjour du souverain. Là où ce ne fut pas le cas, il s'est empressé de les confier, en même temps que d'autres biens non situés sur l'itinéraire royal, à des fidèles de second rang plutôt qu'à l'élite, ou à d'autres membres de sa famille : la dimension politique revient ici au premier plan ${ }^{159}$. Cela n'a pas manqué de susciter un débat. Il revient à Atton de Verceil de l'avoir ouvert, et à Giacomo Vignodelli d'avoir attiré l'attention sur lui ${ }^{160}$. Atton prend l'exemple du gouvernement de Hugues comme repoussoir, c'est-à-dire l'anti-modèle de ce qui arriverait si les Italiens laissaient le roi de Germanie monter sur le trône. Hugues est le type même du dux étranger qui, privé de base bénéficiale solide, se trouve contraint de renverser les équilibres antérieurs pour se créer de toutes pièces un réseau de fidèles qui ne dépendent que de lui. En favorisant ceuxlà plutôt que les milites primi ordinis, il a un usage subversif des biens fiscaux, sans aller pour autant jusqu'au bout de sa logique, qui devrait l'amener à une redistribution générale de la terre publique.

La question qu'il faut alors poser est celle de savoir si les Ottons, après Hugues, ont encore eu la capacité concrète à agir de manière aussi discrétionnaire sur les biens fiscaux ou s'il n'était pas trop tard. Pour en récupérer l'usage, il aurait fallu mener la même politique d'élimination physique des puissants. La reprise en main des biens publics, les uns « gelés » au service exclusif de la royauté, les autres mis en circulation au profit d'homines novi, s'était faite dans un climat de brutalité. Très vite, les biens assignés en douaire à Berthe et à Adélaïde furent au fond la seule réserve sur laquelle purent agir les successeurs de Hugues. Mais désormais, l'enjeu était moins sur le plan foncier que sur celui des droits, donc des revenus : en poursuivant et amplifiant la dévolution des droits de justice à partir des années 960, c'est une fois encore sur le prélèvement indirect que l'on faisait fond.

\footnotetext{
159 Ainsi pour le monastère S. Salvatore d'Agna, confié à Tegrimo, fondateur de la dynastie des comtes Guidi (Schiaparelli, I diplomi di Ugo e di Lotario..., no 9) ; pour Auriola et Foro, cédés au comte Aleramo, et qui font contrepoids à l'influence des marquis d'Ivrée (Schiaparelli, I diplomi di Ugo e di Lotario..., ${ }^{\text {os }} 35$, 53) ; pour Sospiro, dont l'attribution à Rotlinda, fille naturelle de Hugues, profitait à son époux Elisiardus, un fidèle originaire de Provence dépourvu de base matérielle en Italie (Vignodelli, « Berta e Adelaide », p. 268-271 ; voir aussi n 79) ; pour Ronco et la curtis Spoletina, donnée à Milon de Vérone $\left(\mathrm{n}^{\circ} 60\right)$.

${ }^{160}$ G. Vignodelli, « La competizione per i beni fiscali : Ugo di Arles e le aristocrazie del regno italico (926-945)», in G. Bührer-Thierry, R. Le Jan et V. Loré (éd.), Acquérir, prélever, contrôler : les ressources en compétition, 400-1100, Turnhout, 2017 (Haut Moyen Âge, 25), p. 151-169.
} 